DTP 93-29

hep-ph/9308236

\title{
Dynamics of Moving and Spinning Skyrmions
}

\author{
B.J.Schroers* \\ Department of Mathematical Sciences \\ South Road \\ Durham DH1 3LE \\ United Kingdom
}

published in Z.Phys.C61(1994) 479

\begin{abstract}
We study the intermediate- and long-range forces between moving and spinning Skyrmions, employing two different methods. One uses a relativised product ansatz for the Skyrme fields, the other models Skyrmions as triplets of scalar dipoles. The methods lead to the same finite-dimensional Lagrangian dynamical system which may be interpreted as a point-particle approximation to Skyrmion dynamics. We discuss in detail the dynamics in the so-called attractive channel and the interaction between wellseparated and rapidly spinning Skyrmions, and point out the resemblance between the latter and the one-pion exchange potential in nuclear physics.
\end{abstract}

* e-mail: b.j.schroers@durham.ac.uk 


\section{Introduction}

The Skyrme model is a non-linear, non-integrable field theory, defined on $3+1$ dimensional Minkowski space, which has soliton solutions [1]. Here we will use the term "soliton" to mean what is also sometimes called a topological soliton: a localised, finite-energy and smooth solution of the field equations which is stable for topological reasons. Examples include lumps in the $\mathbf{C P}^{n}$ model, vortices in the abelian Higgs model and BPS monopoles in Yang-Mills-Higgs theory. In all cases the solitons have an associated integer winding number which is a topological invariant and which is also called the soliton number. Skyrme solitons of soliton number one are referred to as Skyrmions. Suitably quantised they are models for physical baryons.

Given the long-standing difficulties in finding a satisfactory theoretical model for the interaction of two baryons (the nuclear two-body problem) it is not surprising that much effort has been devoted to the study of the classical and quantised interaction of two Skyrmions. In the absence of integrability one cannot hope to find exact time-dependent solutions which represent twoSkyrmion scattering or bound states, and one therefore has to resort either to numerical methods or to some kind of approximation.

One scheme which has been used to great effect in the study of soliton dynamics at low energy is the adiabatic or moduli space approximation, explained systematically in ref. [2]. In this scheme one views the field theory as an infinite-dimensional Lagrangian dynamical system $(\mathcal{C}, L)$, consisting of a configuration space $\mathcal{C}$, the space of all fields at a given time, and a Lagrangian of the form $L=T-V$, where $T$ is the kinetic energy functional and $V$ is the potential energy functional. One then truncates the theory to a finite-dimensional Lagrangian system by choosing a finite-dimensional submanifold $\mathcal{M} \in \mathcal{C}$ and restricting $L$ to $\mathcal{M}$. The space $\mathcal{M}$ is called the moduli space and should contain all configurations which, on general physical grounds, are deemed to be relevant at low energy. In theories with topological solitons the procedure is somewhat simplified because $\mathcal{C}$ decomposes naturally into disjoint sectors $\mathcal{C}_{n}$ which are each labelled by the winding number $n$ of the fields in it. Correspondingly, one may consider each winding number separately and choose, for given $n$, a truncation $\mathcal{M}_{n}$ of $\mathcal{C}_{n}$. The elements of $\mathcal{M}_{n}$ are called $n$-solitons. The basic assumption of the moduli space approximation is that there exist finite-dimensional manifolds $\mathcal{M}_{n}$ such that the solutions of the truncated Lagrangian systems $\left(\mathcal{M}_{n}, L_{\mid \mathcal{M}_{n}}\right)$ are, in some sense, good approximations to solutions of the full field theory.

How does one find candidates for $\mathcal{M}_{n}$ ? $\mathcal{M}_{1}$ is usually defined as the set of all fields with winding number one which minimise $V$. Typically, this set includes internal as well as position

parameters. For $n \geq 2, \mathcal{M}_{n}$ should include configurations consisting of $n$ well-separated single solitons with arbitrary internal parameters and positions, and one therefore requires that dim $\mathcal{M}_{n}=n \times \operatorname{dim} \mathcal{M}_{1}$. In certain theories, which are said to be of Bogomol'nyi type, this requirement is automatically satisfied if one defines $\mathcal{M}_{n}$, like $\mathcal{M}_{1}$, as the set of fields in $\mathcal{C}_{n}$ which minimise $V$. It 
follows that $V$ is constant on $\mathcal{M}_{n}$, which means physically that there are no static forces between solitons of Bogomol'nyi type. As a result the truncated dynamics is entirely determined by the induced kinetic energy. This is generally of the form $T=\frac{1}{2} g_{i j} \dot{q}_{i} \dot{q}_{j}$, where $\left\{q_{i}\right\}$ are coordinates on $\mathcal{M}_{n}$ and the matrix $g_{i j}$, which depends on $q_{i}$, defines a Riemannian metric on the moduli space. Thus in the moduli space approximation to the slow motion of solitons of Bogomol'nyi type the interactive dynamics of $n$ solitons is modelled by geodesic motion on the moduli space. This is an adiabatic approximation because it approximates the time evolution of $n$-solitons by a sequence of static solutions of the field equations. So far the most interesting and successful application of moduli space approximation has been to two-soliton dynamics in theories of Bogomol'nyi type.

Skyrme's theory is not of Bogomol'nyi type, but the static forces between Skyrme solitons are quite weak. It has therefore been suggested [2] that Skyrme solitons, like Bogomol'nyi type solitons, might be amenable to the moduli space approximation. This requires that one finds suitable moduli spaces for Skyrme's theory. $\mathcal{M}_{1}$, defined as above, is six-dimensional and contains three translational and three rotational degrees of freedom. Thus $\mathcal{M}_{2}$ should be 12 -dimensional. The set of minima of the potential energy $V$ amongst two-Skyrmions is an eight-dimensional manifold of coincident Skyrmions [3] and does not include well-separated Skyrmions. On the other hand, there is a 12-dimensional family of two-Skyrmions, namely the fields of the product form, first considered by Skyrme in ref. [1], which includes well-separated Skyrmions but does not correctly describe the low-energy coincident configurations. In ref. [2] N.S. Manton suggested a method of constructing a 12-dimensional manifold $\mathcal{M}^{12}$ which would include both the coincident and the well-separated Skyrme fields just described. More recently Atiyah and Manton have shown that one can obtain good approximations to Skyrme fields of low energy by calculating the holonomy of $S U(2)$ instantons. They obtain a 12-dimensional manifold $\tilde{\mathcal{M}}^{12}$ of two-Skyrmions in this way and describe its topological and differentiable structure in ref. [5]. They argue further that $\tilde{\mathcal{M}}^{12}$ should be a good approximation to $\mathcal{M}^{12}$. The next step in the moduli space approximation, which has not yet been carried out, is to calculate the induced Lagrangian on $\mathcal{M}^{12}$ or on $\tilde{\mathcal{M}}^{12}$. There are partial results for the potential, but little is known about the kinetic energy.

In the present paper we study the kinetic energy of two well-separated Skyrmions which, statically, are well described by the product ansatz. Physically, this means studying the velocitydependent forces between moving and spinning Skyrmions. According to the moduli space approximation one should, in order to calculate these forces, allow the 12 parameters in the product ansatz to vary with time and express the kinetic energy in terms of the 12 parameters and their derivatives with respect to time. A few of the terms generated in this way were calculated in ref. [6] and a more complete numerical study was undertaken recently [7]. In this paper we will argue that this approach does not lead to the correct effective Lagrangian. Rather, one must allow the individual Skyrmions to Lorentz-contract when they move and modify the product ansatz to take into account this and other relativistic effects. 
To back up our results we re-derive them using a different method. Our second calculation is based on the observation that, from afar, a Skyrmion looks like a triplet of point-like scalar dipoles. It has been known for a long time that the potential between two static Skyrmions can be understood in this way. Using retarded potentials we calculate the Lagrangian for the interaction of two spinning and moving triplets of scalar dipoles including all terms which are at most quadratic in linear and angular velocities. Similar retarded potential calculations, which may be interpreted as point-particle approximations to soliton dynamics, have been carried out for BPS monopoles [8] and maximally charged black holes [9] and have confirmed the moduli space approximation for the long-range dynamics. In our case the Lagrangian for the dipole interaction agrees with the Skyrme Lagrangian evaluated on the relativised product ansatz.

The aim of this paper is two-fold. Specifically we want to understand the importance and nature of kinematic effects in two-Skyrmion dynamics. More generally we address the wider question of how to approximate classical soliton interactions. In this introduction we have described the moduli space approximation in some detail because it provides a coherent and general attempt to answer that question. At the end of this paper we will give a critical assessment of the moduli space approximation in the light of our results.

\section{The Skyrme Model}

The fundamental field of Skyrme's theory is a map

$$
U: \mathrm{M}^{4} \mapsto S U(2)
$$

where $\mathrm{M}^{4}$ is Minkowski space with signature $(+,-,-,-)$. We denote elements of $\mathrm{M}^{4}$ by $x$, with coordinate $x^{\mu}, \mu=0,1,2,3$. Sometimes we also write $x=(t, \boldsymbol{x})$. It is often useful to parametrise $U$ as

$$
U(x)=\frac{1}{f_{\pi}}(\sigma(x)+i \boldsymbol{\pi}(x) \cdot \boldsymbol{\tau})
$$

with the constraint $\sigma^{2}+\boldsymbol{\pi}^{2}=f_{\pi}^{2} \cdot \tau^{a}, a=1,2,3$, are the Pauli matrices, $\pi^{a}$ the pion fields and $f_{\pi}$ is the pion decay constant. The Lagrangian density is best written in terms of the pull-back of the left-invariant form $U^{\dagger} d U$

$$
L_{\mu}=U^{\dagger} \partial_{\mu} U
$$

where $\partial_{\mu}=\partial / \partial x^{\mu}$, or in terms of the right-invariant analogue

$$
R_{\mu}=U \partial_{\mu} U^{\dagger}
$$

It reads

$$
\begin{aligned}
\mathcal{L} & =-\frac{f_{\pi}^{2}}{4} \operatorname{tr}\left(L_{\mu} L^{\mu}\right)+\frac{1}{32 e^{2}} \operatorname{tr}\left(\left[L_{\mu}, L_{\nu}\right]\left[L^{\mu}, L^{\nu}\right]\right) \\
& =-\frac{f_{\pi}^{2}}{4} \operatorname{tr}\left(R_{\mu} R^{\mu}\right)+\frac{1}{32 e^{2}} \operatorname{tr}\left(\left[R_{\mu}, R_{\nu}\right]\left[R^{\mu}, R^{\nu}\right]\right) .
\end{aligned}
$$


The constant $e$ is a free parameter of the Skyrme model and it is customary to treat $f_{\pi}$ as a free parameter as well. We will adjust $e$ and $f_{\pi}$ later to fit experimental data. We fix our units by setting Planck's constant and the speed of light to 1 .

Note the large symmetry group of the action corresponding to (2.5). It is invariant under

$$
\text { Poincaré group } \times S O(4)_{\text {chiral }}
$$

where

$$
S O(4)_{\text {chiral }} \cong \frac{S U(2) \times S U(2)}{\mathbf{Z}_{2}}
$$

acts on $U(x)$ via left and right multiplication with constant $S U(2)$ matrices. The Euler-Lagrange equations take the form of conservation laws: the currents

$$
\bar{L}_{\mu}=L_{\mu}+\frac{1}{4 e^{2} f_{\pi}^{2}}\left[L_{\nu},\left[L^{\nu}, L_{\mu}\right]\right]
$$

and

$$
\bar{R}_{\mu}=R_{\mu}+\frac{1}{4 e^{2} f_{\pi}^{2}}\left[R_{\nu},\left[R^{\nu}, R_{\mu}\right]\right]
$$

satisfy

$$
\partial_{\mu} \bar{L}^{\mu}=\partial_{\mu} \bar{R}^{\mu}=0
$$

So far we have assumed that the pions are massless. Physical pions have a small mass of about $m_{\pi} \approx 140 \mathrm{MeV}$ (we ignore the mass difference between $\pi^{0}$ and $\pi^{ \pm}$). This can be incorporated into the Skyrme model by adding

$$
\frac{1}{4} f_{\pi}^{2} m_{\pi}^{2} \operatorname{tr}\left(U+U^{\dagger}-2\right)
$$

to the Lagrangian. This term explicitly breaks the chiral symmetry to $S O(3)_{\text {isospin }}$, which acts via conjugation with a constant $S U(2)$ element $A$

$$
U(x) \mapsto A U(x) A^{\dagger}
$$

or alternatively, in terms of the associated orthogonal matrix

$$
\mathcal{A}^{a b}=\frac{1}{2} \operatorname{tr}\left(\tau^{a} A \tau^{b} A^{\dagger}\right)
$$

and the pion field,

$$
\pi^{a}(x) \rightarrow \mathcal{A}^{a b} \pi^{b}(x) .
$$


In the presence of the pion mass term the equations of motion become

$$
\partial_{\mu} \bar{L}^{\mu}=-\partial_{\mu} \bar{R}^{\mu}=\frac{1}{2} m_{\pi}^{2}\left(U-U^{\dagger}\right) .
$$

It turns out that the predictions of the Skyrme model for the static properties of a single nucleon do not depend much on the pion mass, as long as it is small [13]. For the interaction of two Skyrme solitons, however, the pion mass has important physical consequences. Here we will carry out calculations mainly for the case $m_{\pi}=0$ where the theory is more symmetric and the mathematical manipulations somewhat simpler. In certain applications we can then take into account the physical pion mass by general physical arguments.

Viewed as an infinite dimensional Lagrangian system the Skyrme model's configuration space $\mathcal{C}$ is the space of maps

$$
U: \mathbf{R}^{3} \mapsto S U(2) .
$$

The Lagrangian $L=\int d^{3} x \mathcal{L}$ has the usual form

$$
L=T-V
$$

where $T$ is the kinetic energy

$$
T=-\frac{f_{\pi}^{2}}{4} \operatorname{tr}\left(L_{0} L_{0}\right)-\frac{1}{16 e^{2}} \operatorname{tr}\left(\left[L_{0}, L_{i}\right]\left[L_{0}, L_{i}\right]\right)
$$

and

$$
V=-\frac{f_{\pi}^{2}}{4} \operatorname{tr}\left(L_{i} L_{i}\right)-\frac{1}{32 e^{2}} \operatorname{tr}\left(\left[L_{j}, L_{i}\right]\left[L_{j}, L_{i}\right]\right)
$$

is the potential energy functional. To make $V$ well defined, one needs to impose a finite energy requirement on elements of $\mathcal{C}$. In practice one demands that the field $U$ tends to a constant matrix, which one usually takes to be the identity, at spatial infinity. This allows one to compactify space to $S^{3}$ and regard $U$ as an element of $\Pi_{3}\left(S^{3}\right) \cong \mathbf{Z}$ with an associated integer degree or winding number. This number is absolutely conserved during time evolution and is identified with the baryon number $B$. A subset of $\mathcal{C}$ whose elements have a definite winding number $B$ is denoted by $\mathcal{C}_{B}$.

The imposition of the asymptotic condition also has important consequences for the symmetry of the theory. The chiral symmetry is broken to $S O(3)_{\text {isospin }}$. The euclidean group $E_{3}=S O(3)_{\text {space }} \times$ $\mathbf{R}^{3}$, whose elements $(\mathcal{G}, \boldsymbol{X})$ act on fields $U \in \mathcal{C}$ via pullback:

$$
U(\boldsymbol{x}) \mapsto U\left(\mathcal{G}^{-1}(\boldsymbol{x}-\boldsymbol{X})\right),
$$

and the isopspin rotations $S O(3)_{\text {isospin }}$ are symmetries of the Lagrangian and map each sector $\mathcal{C}_{B}$ of the configuration space into itself. There is also a discrete symmetry, the combination of parity 
operations in space and isospace. Separately these parity operations reverse the sign of the winding number $B$, but the map

$$
\Pi: U(\boldsymbol{x}) \mapsto U^{\dagger}(-\boldsymbol{x})
$$

leaves $B$ unchanged. Thus for each baryon number $B$ the symmetry group of the Lagrangian system $\left(\mathcal{C}_{B}, L_{\mid \mathcal{C}_{B}}\right)$ is

$$
S=\Pi \times E_{3} \times S O(3)_{\text {isospin }}
$$

\section{Dynamics of a Single Skyrmion}

We briefly review the standard approximations used in discussing single Skyrmion dynamics and assess their validity.

The usual way to obtain a particular solution of the static field equations in the sector $B=1$ is to make the hedgehog ansatz

$$
U_{H}(\boldsymbol{x})=\exp (i f(r) \hat{\boldsymbol{x}} \cdot \boldsymbol{\tau}) \quad r=|\boldsymbol{x}|, \quad \hat{\boldsymbol{x}}=\frac{\boldsymbol{x}}{r}
$$

with the boundary conditions $f(0)=\pi$ and $f(\infty)=0$ to ensure that $U_{H}$ has winding number 1 . Minimising the potential energy $V$ restricted to Skyrme fields of hedgehog type leads to a second order ordinary differential for $f$, given in ref. [10], which can easily be solved numerically. In the following $f$ will always stand for that solution and the corresponding field $U_{H}$ will be referred to as the standard Skyrmion. We are particularly interested in the behaviour of $f$ for large $r$, which is

$$
f \sim \frac{\lambda}{r^{2}}+O\left(\frac{1}{r^{8}}\right)
$$

where $\lambda$ is numerically found to be $2.16 / e^{2} f_{\pi}^{2}$. There is strong numerical evidence that the standard Skyrmion is also a minimum of $V$ among all fields in the $B=1$ sector and that all minima in this sector can be obtained by acting on the standard Skyrmion with the symmetry group $S$. This group is 9-dimensional, but when it acts on a field of the hedgehog form spatial and isospin rotations are equivalent. Hence the orbit of the standard Skyrmion under $S$ is a 6dimensional manifold which we denote by $\mathcal{M}_{1}$. This is the manifold which is used as the moduli space in the $B=1$ sector. It is diffeomorphic to $\mathbf{R}^{3} \times S O(3)$ and therefore its elements, which are called Skyrmions, are fully specified by their position and orientation. On $\mathcal{M}_{1}$ the potential energy is constant and equal to the Skyrmion's rest mass $M$, which can be calculated from the radial function $f$. The result is $M=73 f_{\pi} / e$ [10]. Thus the induced dynamics is determined by the kinetic energy, which can be found by inserting the ansatz

$$
A(t) U(\boldsymbol{x}-\boldsymbol{X}(t)) A^{\dagger}(t)
$$


into (2.18). After a lengthy calculation one finds the induced Lagrangian [10]

$$
L_{1}=-M+\Lambda \operatorname{tr} \dot{A} \dot{A}^{\dagger}+\frac{1}{2} M \dot{\boldsymbol{X}}^{2}
$$

where the dot denotes differentiation with respect to $t$. The moment of inertia $\Lambda$ is also calculated in ref. 10 (where $\Lambda$ is denoted by $\lambda$ ) and found to be $53.3 / e^{3} f_{\pi}$. The angular velocity $\boldsymbol{\alpha}$ is usually defined via $A^{\dagger} \dot{A}=-\frac{i}{2} \boldsymbol{\alpha} \cdot \boldsymbol{\tau}$. In terms of the $S O(3)$ matrix $\mathcal{A}$ associated to $A$ one can also write $\mathcal{A}^{-1} \dot{\mathcal{A}}=\boldsymbol{\alpha} \cdot \boldsymbol{s}$ where $s_{i}, i=1,2,3$ are the generators of the $S O(3)$ Lie algebra satisfying $\left[s_{i}, s_{j}\right]=\epsilon_{i j k} s_{k}$. In terms of $\boldsymbol{\alpha}, \Lambda \operatorname{tr} \dot{A} \dot{A}^{\dagger}=\frac{1}{2} \Lambda \boldsymbol{\alpha}^{2}$. According to the equations of motion derived from $L_{1}$ a Skyrmion moves uniformly in space and rotates rigidly at constant angular velocity.

So far the standard, approximate treatment of the $B=1$ sector. How useful are the approximations made so far? Clearly the uniformly moving but non-rotating solution is a good approximation to the exact solution one obtains by Lorentz-boosting a static Skyrmion, provided the speed is small and Lorentz contraction can be neglected. However, in this case the moduli space approximation is merely a complicated way of deriving something trivial. The other, rigidly rotating solution found in the moduli space approximation is almost certainly a poor approximation to solutions of the full field equations. This can be seen in a number of ways. One argument, frequently discussed in the literature and reviewed in ref. [11], is based on the observation that there are centrifugal effects arising from the rotation which could pull the Skyrmion apart. We prefer to discuss the rotating Skyrmion from another point of view which suggests that an exact solution exists only if $m_{\pi} \neq 0$ and that it is not of the hedgehog form at all. Our discussion is based on the following simple observations. Far away from the centre of the Skyrmion the field equations (2.10) simplify to three uncoupled free scalar wave equations for the massless pion fields

$$
\frac{\partial^{2} \pi^{a}}{\partial t^{2}}-\Delta \pi^{a}=0 \quad a=1,2,3
$$

Furthermore the pion fields of a Skyrmion $A U_{H}(\boldsymbol{x}) A^{\dagger}$ centred at the origin behave asymptotically like

$$
\pi^{a} \sim f_{\pi} \lambda \frac{\mathcal{A}^{a j} \hat{x}^{j}}{r^{2}}=f_{\pi} \lambda \frac{\mathcal{A}^{i j} e_{a}^{i} \hat{x}^{j}}{r^{2}}
$$

where $\left\{\boldsymbol{e}_{a}\right\}$ is the standard basis of $\mathbf{R}^{3}$. Defining

$$
\boldsymbol{p}_{a}=4 \pi f_{\pi} \lambda \mathcal{A}^{-1} \boldsymbol{e}_{a} \quad\left(\text { so that } \quad \pi^{a} \sim \frac{\boldsymbol{p}_{a} \cdot \hat{\boldsymbol{x}}}{4 \pi r^{2}}\right)
$$

we see that for sufficiently large $r$ each of the pion fields $\pi^{a}$ can be approximated by a static solution of the scalar wave equation with a suitable (time-independent) dipole source term (we will discuss the charge distribution for a scalar dipole systematically in sect. 6):

$$
\frac{\partial^{2} \phi}{\partial t^{2}}-\Delta \phi=-\boldsymbol{p} \cdot \nabla \delta^{(3)}(\boldsymbol{x})
$$


This suggests that the asymptotic behaviour of each pion field of a rotating Skyrmion should be described by solutions of (3.8) with rotating dipole moments $\boldsymbol{p}_{a}$. More precisely, for a dipole moment $\boldsymbol{p}(t)$ rotating at constant angular velocity $\boldsymbol{\alpha}$, i.e. $\dot{\boldsymbol{p}}(t)=\boldsymbol{p}(t) \times \boldsymbol{\alpha}$, equation (3.8) is solved by

$$
\begin{array}{r}
\phi(t, \boldsymbol{x})=\frac{1}{4 \pi r^{2}}\left[\left(\hat{\boldsymbol{x}} \cdot \boldsymbol{p}_{\|}(t)+(\cos \alpha r+\alpha r \sin \alpha r) \hat{\boldsymbol{x}} \cdot \boldsymbol{p}_{\perp}(t)\right.\right. \\
+(\alpha r \cos \alpha r-\sin \alpha r) \hat{\boldsymbol{x}} \cdot(\boldsymbol{p}(t) \times \hat{\boldsymbol{\alpha}})]
\end{array}
$$

where $\alpha=|\boldsymbol{\alpha}|$ and $\boldsymbol{p}_{\|}$and $\boldsymbol{p}_{\perp}$ are the components of $\boldsymbol{p}$ parallel and perpendicular to $\boldsymbol{\alpha}$. This should be compared with the asymptotic pion fields of the rigidly rotating Skyrmion

$$
\pi^{a}(t, \boldsymbol{x}) \sim \frac{\boldsymbol{p}_{a}(t) \cdot \hat{\boldsymbol{x}}}{4 \pi r^{2}}
$$

Clearly the rigidly rotating solution is only adequate if $r<1 / \alpha$. Furthermore we calculate, using standard formulae from radiation theory, that the exact solution (3.9) will radiate away its energy at a rate

$$
P=\frac{1}{12 \pi} \alpha^{4}|\boldsymbol{p}|^{2}
$$

This suggests that a spinning Skyrmion should similarly lose energy through radiation and we can use the formula (3.11) to estimate the time for which the approximation of uniform rotation is good. To sum up, the dipole picture indicates that, for $m_{\pi}=0$, a rigidly and uniformly rotating Skyrme field can only be a good approximation to an exact solution of the Skyrme field equations for times less than

$$
t_{\text {life }}=\frac{\frac{1}{2} \Lambda \alpha^{2}}{P}=\frac{6 \pi \Lambda}{|\boldsymbol{p}|^{2} \alpha^{2}}
$$

and for distances from the centre of the Skyrmion less than

$$
r_{\max }=\frac{1}{\alpha}
$$

Which value should we insert for the frequency $\alpha$ in order to obtain numerical estimates for the above parameters? Spin 1/2 quantum states of a single Skyrmion are models for physical baryons and spin $3 / 2$ states model the $\Delta$ - resonance. We fix the constants $f_{\pi}$ and $e$ as in ref. [10] to fit the nucleon and $\Delta$ masses

$$
f_{\pi}=65 \mathrm{MeV} \quad \text { and } \quad e=5.45,
$$

so that $f_{\pi}$ is $30 \%$ smaller than its usual value of $93 \mathrm{MeV}$. Thus the frequency at which a classical Skyrmion would have to rotate to have the proton's or neutron's angular momentum is

$$
\alpha_{N} \approx \frac{1}{2 \Lambda} \approx 1.5 f_{\pi} \approx 100 \mathrm{MeV}
$$


We then find that

$$
t_{\text {life }} \approx \frac{3}{f_{\pi}} \approx 3 \times 10^{-23} s \quad \text { and } \quad r_{\max } \approx \frac{0.7}{f_{\pi}} \approx 2 \text { fermi. }
$$

A classical Skyrmion spinning at the frequency $\alpha_{N}$ would therefore radiate away its rotational energy in a very short time if $m_{\pi}=0$, but it could be stabilised by including the physical pion mass since $\alpha_{N}<m_{\pi}$. Note, however, that in a fully quantised form of the Skyrme model the short classical lifetime need not necessarily be a cause for concern: a proton or neutron could only lower its rotational energy by emitting a particle of spin $1 / 2$, but there is no such particle in the model.

The value $r_{\max }$ should be compared with the root-mean-square radius of the Skyrmion's mass distribution, which is $r=0.7$ fermi [11]. The form of the pion fields at $r>2$ fermi does not matter much in the calculation of the static nucleon properties in the Skyrme model, but it is crucial for the long-range interaction of two Skyrmions. In studying the long-range forces between spinning Skyrmions, and in particular in applying the Skyrme model to the nuclear two-body problem, one therefore needs to bear in mind that, no matter whether $m_{\pi}=0$ or $m_{\pi} \neq 0$, the long-range fields of a spinning Skyrmion are not of the hedgehog form.

Even if we restrict ourselves to circumstances where the rigidly rotating hedgehog field is an adequate approximation it does not follow that a moving and spinning Skyrmion is well described by (3.3). Rather, one should Lorentz-boost a stationary and rigidly rotating hedgehog field to obtain a moving and spinning Skyrmion. More precisely, suppose that the Skyrmion is moving with velocity $\boldsymbol{u}$ and has position $\boldsymbol{X}$ at time $t$. Its orientation at time $t$ is given by an $S U(2)$ matrix $A$, and the orientation then changes uniformly with respect to the rest frame time, which we denote by $t^{\prime}$. We set $t^{\prime}=0$ at the space-time point with laboratory coordinates $(t, \boldsymbol{X})$; at a later laboratory time $t+\delta t$, it is given by

$$
t^{\prime}=\gamma(\delta t-\boldsymbol{u} \cdot(\boldsymbol{x}-\boldsymbol{X}))
$$

where $\gamma=\left(1-\boldsymbol{u}^{2}\right)^{-1 / 2}$. Thus the field for a moving and spinning Skyrmion is

$$
U(t+\delta t, \boldsymbol{x})=A\left(t^{\prime}\right) U_{H}\left(\boldsymbol{x}^{\prime}\right) A^{\dagger}\left(t^{\prime}\right),
$$

where

$$
\boldsymbol{x}_{\|}^{\prime}=\gamma\left(\boldsymbol{x}_{\|}-\boldsymbol{X}_{\|}-\boldsymbol{u} \delta t\right) \quad \text { and } \quad \boldsymbol{x}_{\perp}^{\prime}=\boldsymbol{x}_{\perp}-\boldsymbol{X}_{\perp} .
$$

Here the suffixes $\|$ and $\perp$ denote the components parallel and perpendicular to the velocity vector $\boldsymbol{u}$ and $A\left(t^{\prime}\right)$ is an $S U(2)$-valued function defined by

$$
A(0)=A \quad \text { and } \quad-\frac{i}{2} \boldsymbol{\alpha} \cdot \boldsymbol{\tau}=A^{\dagger}\left(t^{\prime}\right) \frac{d A\left(t^{\prime}\right)}{d t^{\prime}} .
$$

where $\boldsymbol{\alpha}$ is a constant vector, representing the angular velocity in the Skyrmion's rest frame. The Skyrme field (3.18) takes into account Lorentz contraction of a moving Skyrmion and the 
relativity of time: if the pion fields' rotation is synchronised in the Skyrmion's rest frame then their rotation will be retarded or advanced in the laboratory frame, depending on their distance from the Skyrmion's centre projected onto $\boldsymbol{u}$.

If the velocity $\boldsymbol{u}$ is constant the field (3.18) is a good description of a spinning and moving Skyrmion for all laboratory times $t+\delta t$. Later in this paper we will consider Skyrmions which do not move uniformly but whose acceleration is small. Then one needs to perform different Lorentz boosts for different laboratory times and the formula (3.18) is only an approximation, valid for small increments $\delta t$. This is sufficient for us, since we will only require Skyrme fields and their rate of change (with respect to laboratory time) at a given laboratory time $t$. The position vector $\boldsymbol{X}$ and the orientation matrix $A$ strictly depend on $t$ but, for ease of notation, we will not write this dependence explicitly. Note also that differentiation with respect to time should be carried out according to

$$
\frac{\partial U}{\partial t}(t, \boldsymbol{x})=\left.\frac{\partial U(t+\delta t, \boldsymbol{x})}{\partial \delta t}\right|_{\delta t=0} .
$$

In our calculations will only take into account the velocity dependence of the field (3.18) to leading order. To this accuracy, and with the limitations discussed earlier, we will make the approximation

$$
t^{\prime} \approx \delta t-\boldsymbol{u} \cdot(\boldsymbol{x}-\boldsymbol{X})
$$

and describe a single moving and spinning Skyrmion by the following field:

$$
U(t+\delta t, \boldsymbol{x})=A\left(t^{\prime}\right) U_{H}(\boldsymbol{\xi}) A^{\dagger}\left(t^{\prime}\right)
$$

where

$$
\boldsymbol{\xi}=\boldsymbol{x}-\boldsymbol{X}-\boldsymbol{u} \delta t+\frac{1}{2}(\boldsymbol{u} \cdot(\boldsymbol{x}-\boldsymbol{X})) \boldsymbol{u}
$$

and it is implied that we use the approximation

$$
A\left(t^{\prime}\right) \approx A\left(\mathbf{1}_{2}-\frac{i}{2} \boldsymbol{\alpha} \cdot \boldsymbol{\tau}(\delta t-\boldsymbol{u} \cdot(\boldsymbol{x}-\boldsymbol{X}))\right) .
$$

When evaluating the Skyrme Lagrangian on fields of this form we only retain terms which are at most quadratic in linear and angular velocities. Remarkably, the evaluation of the Skyrme Lagrangian on the field (3.22) again yields again the Lagrangian $L_{1}$, but now the angular velocity $\boldsymbol{\alpha}$ should be interpreted as the angular velocity in the rest frame (3.19).

For later use we introduce the momenta

$$
\boldsymbol{J}=\Lambda \boldsymbol{\alpha} \quad \text { and } \quad \boldsymbol{I}=\Lambda \mathcal{A} \boldsymbol{\alpha}
$$

which are the conserved quantities due to the invariance of $L_{1}$ under right-translations $A \mapsto A F$ and left-translations $A \mapsto F A$ by a constant $S U(2)$ matrix $F$ respectively. In the usual quantisation of a single Skyrmion $\boldsymbol{J}$ becomes the spin operator and $\boldsymbol{I}$ becomes the isospin operator. 


\section{The Product Ansatz for Moving and Spinning Skyrmions}

In this section we outline our method for calculating the asymptotic form of the Skyrme Lagrangian when evaluated on fields of the product form. This method applies equally to the standard product ansatz, which employs fields of the form (3.3) for the individual Skyrme fields, and to our relativised product ansatz, which uses the fields (3.22). For definiteness, let us consider the latter.

Assume that, at time $t$, one Skyrmion has orientation $A \in S U(2)$, angular velocity in its rest frame $\boldsymbol{\alpha}$, position $\boldsymbol{X}$, and velocity $\boldsymbol{u}$ and the other Skyrmion has orientation $B \in S U(2)$, angular velocity in its rest frame $\boldsymbol{\beta}$, position $\boldsymbol{Y}$ and velocity $\boldsymbol{v}$. Then we write the relativised product ansatz at time $t+\delta t$ close to $t$ for the field describing this situation as

$$
U(t+\delta t, \boldsymbol{x})=U^{(1)}(t+\delta t, \boldsymbol{x}) U^{(2)}(t+\delta t, \boldsymbol{x}) .
$$

Here $U^{(1)}$ is given by (3.22) and $U^{(2)}$ analogously by

$$
U^{(2)}(t+\delta t, \boldsymbol{x})=B\left(t^{\prime \prime}\right) U_{H}(\boldsymbol{\eta}) B^{\dagger}\left(t^{\prime \prime}\right)
$$

where

$$
\begin{aligned}
t^{\prime \prime} & =\left(1-\boldsymbol{v}^{2}\right)^{-\frac{1}{2}}(\delta t-\boldsymbol{v} \cdot(\boldsymbol{x}-\boldsymbol{Y})) \approx \delta t-\boldsymbol{v} \cdot(\boldsymbol{x}-\boldsymbol{Y}) \\
\boldsymbol{\eta} & =\boldsymbol{x}-\boldsymbol{Y}-\boldsymbol{v} \delta t+\frac{1}{2}(\boldsymbol{v} \cdot(\boldsymbol{x}-\boldsymbol{Y})) \boldsymbol{v}
\end{aligned}
$$

and the function $B\left(t^{\prime \prime}\right)$ is approximated by the following expression, adequate for our purposes:

$$
B\left(t^{\prime \prime}\right) \approx B\left(\mathbf{1}_{2}-\frac{i}{2} \boldsymbol{\beta} \cdot \boldsymbol{\tau}(\delta t-\boldsymbol{v} \cdot(\boldsymbol{x}-\boldsymbol{Y}))\right)
$$

When inserting the field (4.1) into the Skyrme Lagrangian we first carry out all necessary differentiations and then set $\delta t=0$. For our calculations it will also be useful to introduce

$$
\boldsymbol{R}=\boldsymbol{X}-\boldsymbol{Y}, R=|\boldsymbol{R}|
$$

for the relative position. The computation of the Lagrangian is simplified by the observation that

$$
L_{\mu}=U^{\dagger} \partial_{\mu} U=U^{(2) \dagger}\left(L_{\mu}^{(1)}-R_{\mu}^{(2)}\right) U^{(2)}
$$

where $L_{\mu}^{(1)}=U^{(1) \dagger} \partial_{\mu} U^{(1)}$ and $R_{\mu}^{(2)}=U^{(2)} \partial_{\mu} U^{(2) \dagger}$. When inserting this expression into (2.5) the conjugation with $U^{(2)}$ drops out. One still generates a large number of terms, but they can be further classified according to their degree in $L_{\mu}^{(1)}$ or $R_{\mu}^{(2)}$. The terms which involve only either $L_{\mu}^{(1)}$ or $R_{\mu}^{(2)}$ give the sum of the Lagrangians for a single free Skyrmion. When expanding the

remaining terms in the Lagrangian in powers of $1 / R$, terms which are linear in $L_{\mu}^{(1)}$ or $R_{\mu}^{(2)}$ give 
the leading contribution. Neglecting the terms which are quadratic in both $L_{\mu}^{(1)}$ and $R_{\mu}^{(2)}$ we get

$$
\begin{aligned}
\mathcal{L} & =-\frac{f_{\pi}^{2}}{4} \operatorname{tr}\left(L_{\mu}^{(1)} L^{(1) \mu}\right)+\frac{1}{32 e^{2}} \operatorname{tr}\left(\left[L_{\mu}^{(1)}, L_{\nu}^{(1)}\right]\left[L^{(1) \mu}, L^{(1) \nu}\right]\right) \\
& -\frac{f_{\pi}^{2}}{4} \operatorname{tr}\left(R_{\mu}^{(2)} R^{(2) \mu}\right)+\frac{1}{32 e^{2}} \operatorname{tr}\left(\left[R_{\mu}^{(2)}, R_{\nu}^{(2)}\right]\left[R^{(2) \mu}, R^{(2) \nu}\right]\right) \\
& +\frac{f_{\pi}^{2}}{2} \operatorname{tr}\left(L_{\mu}^{(1)} \bar{R}^{(2) \mu}+\bar{L}_{\mu}^{(1)} R^{(2) \mu}-L_{\mu}^{(1)} R^{(2) \mu}\right) .
\end{aligned}
$$

In terms of the mass $M$ and the moment of inertia $\Lambda$ of a single hedgehog Skyrmion as defined earlier (3.4) we can write the Lagrangian restricted to fields of the product form (4.1)

$$
L_{2}=-2 M+\frac{1}{2} M \boldsymbol{u}^{2}+\frac{1}{2} \Lambda \boldsymbol{\alpha}^{2}+\frac{1}{2} M \boldsymbol{v}^{2}+\frac{1}{2} \Lambda \boldsymbol{\beta}^{2}+L_{\mathrm{int}}
$$

where

$$
L_{\mathrm{int}}=\frac{f_{\pi}^{2}}{2} \int d^{3} x \operatorname{tr}\left(L_{\mu}^{(1)} \bar{R}^{(2) \mu}+\bar{L}_{\mu}^{(1)} R^{(2) \mu}-L_{\mu}^{(1)} R^{(2) \mu}\right)
$$

Our aim is to calculate the leading terms in an expansion of $L_{\text {int }}$ in powers of $1 / R$. Our calculations are based on the idea of treating the field of one soliton as constant in the vicinity of the other. In order to evaluate the integral (4.8) we divide the region of integration into three parts

$$
\mathbf{R}^{3}=B_{\rho}(\boldsymbol{X}) \cup B_{\rho}(\boldsymbol{Y}) \cup V_{\rho}(\boldsymbol{X}, \boldsymbol{Y})
$$

where $B_{\rho}(\boldsymbol{X})$ is the ball of radius $\rho$ around $\boldsymbol{X}$ and

$$
V_{\rho}(\boldsymbol{X}, \boldsymbol{Y})=\mathbf{R}^{3}-\left(B_{\rho}(\boldsymbol{X}) \cup B_{\rho}(\boldsymbol{Y})\right)
$$

With this division we can exploit the simple asymptotic form of the hedgehog profile. Equation (3.2) implies that, for large $|\boldsymbol{\xi}|$,

$$
L_{\mu}^{(1)}(t, \boldsymbol{x}) \sim l_{\mu}^{(1)}(t, \boldsymbol{x}):=i \lambda \partial_{\mu}\left(A\left(t^{\prime}\right) \frac{\hat{\boldsymbol{\xi}} \cdot \boldsymbol{\tau}}{|\boldsymbol{\xi}|^{2}} A^{\dagger}\left(t^{\prime}\right)\right)_{\mid \delta t=0}
$$

and similarly for large $|\boldsymbol{\eta}|$

$$
R_{\mu}^{(2)}(t, \boldsymbol{x}) \sim r_{\mu}^{(2)}(t, \boldsymbol{x}):=-i \lambda \partial_{\mu}\left(B\left(t^{\prime \prime}\right) \frac{\hat{\boldsymbol{\eta}} \cdot \boldsymbol{\tau}}{|\boldsymbol{\eta}|^{2}} B^{\dagger}\left(t^{\prime \prime}\right)\right)_{\mid \delta t=0} .
$$

Since $L_{\mu}^{(1)}$ and $\bar{L}_{\mu}^{(1)}$ differ only by a triple product of the $L_{\mu}^{(1)}$ 's (similarly for $R_{\mu}^{(2)}$ ) we also have, in the above limits,

$$
\bar{L}_{\mu}^{(1)} \sim l_{\mu}^{(1)} \quad \text { and } \quad \bar{R}_{\mu}^{(2)} \sim r_{\mu}^{(2)}
$$

As $R$ becomes large we will choose $\rho$ so large that outside $B_{\rho}(\boldsymbol{X})$ and $B_{\rho}(\boldsymbol{Y})$ we can replace $L_{\mu}^{(1)}$ and $\bar{L}_{\mu}^{(1)}$ by $l_{\mu}^{(1)}$, as well as $R_{\mu}^{(2)}$ and $\bar{R}_{\mu}^{(2)}$ by $r_{\mu}^{(2)}$. But we also want to keep $\rho$ small enough so that 
$l_{\mu}^{(1)}$ and $r_{\mu}^{(2)}$ may be considered constant over $B_{\rho}(\boldsymbol{Y})$ and $B_{\rho}(\boldsymbol{X})$ respectively. We achieve this by letting $\rho \rightarrow \infty$ as $R \rightarrow \infty$ in such a way that $\rho / R \rightarrow 0$. We then find

$$
\begin{aligned}
L_{\text {int }} & \sim \frac{f_{\pi}^{2}}{2}\left[\operatorname{tr}\left(r_{\mu}^{(2)}(\boldsymbol{X}) \int_{B_{\rho}(\boldsymbol{X})} d^{3} x \bar{L}^{(1) \mu}\right)+\operatorname{tr}\left(l_{\mu}^{(1)}(\boldsymbol{Y}) \int_{B_{\rho}(\boldsymbol{Y})} d^{3} x \bar{R}^{(2) \mu}\right)\right. \\
& \left.+\int_{V_{\rho}(\boldsymbol{X}, \boldsymbol{Y})} \operatorname{tr}\left(l_{\mu}^{(1)} r^{(2) \mu}\right)\right] .
\end{aligned}
$$

This formula is the starting point in most of our calculations in the following sections.

\section{The Two-Skyrmion Potential Revisited}

As a first step we consider the static situation, where $\boldsymbol{u}=\boldsymbol{v}=0$ and $A\left(t^{\prime}\right)=A$ and $B\left(t^{\prime \prime}\right)=B$ are constant $S U(2)$ matrices. Then $-L_{\text {int }}$ reduces to the two-Skyrmion potential $V_{2}$ :

$$
\begin{aligned}
V_{2} & =\frac{f_{\pi}^{2}}{2} \int d^{3} x \operatorname{tr}\left(L_{i}^{(1)} \bar{R}_{i}^{(2)}+\bar{L}_{i}^{(1)} R_{i}^{(2)}-L_{i}^{(1)} R_{i}^{(2)}\right) \\
& \sim \frac{f_{\pi}^{2}}{2}\left[\operatorname{tr}\left(r_{i}^{(2)}(\boldsymbol{X}) \int_{B_{\rho}(\boldsymbol{X})} d^{3} x\left(\bar{L}_{i}^{(1)}-l_{i}^{(1)}\right)\right)+\operatorname{tr}\left(l_{i}^{(1)}(\boldsymbol{Y}) \int_{B_{\rho}(\boldsymbol{Y})} d^{3} x\left(\bar{R}_{i}^{(2)}-r_{i}^{(2)}\right)\right)\right. \\
& \left.+\int_{\mathbf{R}^{3}} d^{3} x \operatorname{tr}\left(l_{i}^{(1)} r_{i}^{(2)}\right)\right] .
\end{aligned}
$$

The asymptotic form of $V_{2}$ was already given by Skyrme in ref. [4] and the potential energy of static product fields for any value of $R$ was studied numerically in ref. [12]. We will re-derive Skyrme's result using a different method which we find mathematically more satisfactory. In the course of our calculation we will also derive a number of formulae which we will need in the remainder of the paper.

First we introduce the $S O(3)$ matrix

$$
\mathcal{O}^{i j}=\frac{1}{2} \operatorname{tr}\left(\tau^{i} A^{\dagger} B \tau^{j} B^{\dagger} A\right)
$$

specifying the relative orientation of the two Skyrmions. The main input of our calculation is the fact that both $U^{(1)}$ and $U^{(2)}$ satisfy the Euler-Lagrange equation for static fields:

$$
\partial_{i} \bar{L}_{i}^{(1)}=\partial_{i} \bar{R}_{i}^{(2)}=0 .
$$

Let us for the moment concentrate on $\bar{L}_{i}^{(1)}$ and write it as

$$
\bar{L}_{i}^{(1)}=i A \bar{L}_{i m} \tau_{m} A^{\dagger} .
$$

Then, for each $m=1,2,3, \bar{L}_{i m}$ are the components of a smooth vector field on $\mathbf{R}^{3}$ and (5.3) implies that this vector field is divergenceless. Thus, for each $m$ we can find a vector field with components $Z_{k m}$ such that

$$
\bar{L}_{i m}=\epsilon_{i j k} \partial_{j} Z_{k m} .
$$


Explicitly we find

$$
Z_{k m}(\boldsymbol{x}+\boldsymbol{X})=\left(\sin ^{2} f(r)-\frac{1}{e^{2} f_{\pi}^{2}} \frac{\sin ^{4} f(r)}{r^{2}}\right) \hat{x}_{k} \hat{x}_{m}+\left(\frac{1}{2} r f^{\prime}(r)+\frac{1}{e^{2} f_{\pi}^{2}} f^{\prime}(r) \frac{\sin ^{2} f(r)}{r}\right) \epsilon_{k m n} \hat{x}_{n} .
$$

Hence

$$
\begin{aligned}
\int_{B_{\rho}(\boldsymbol{X})} d^{3} x \bar{L}_{i}^{(1)}(\boldsymbol{x}) & =i A \tau_{m} A^{\dagger} \int_{\partial B_{\rho}(0)} d S \epsilon_{i j k} Z_{k m}(\boldsymbol{x}+\boldsymbol{X}) \hat{x}_{j} \\
& =i A \tau_{m} A^{\dagger} \int_{\partial B_{\rho}(0)} d S\left(\left(\delta_{i m}-\hat{x}_{i} \hat{x}_{m}\right)\left(\frac{1}{2} r f^{\prime}(r)+\frac{1}{e^{2} f_{\pi}^{2}} f^{\prime}(r) \frac{\sin ^{2} f(r)}{r}\right)\right) \\
& =i A \tau_{i} A^{\dagger} \frac{4 \pi}{3}\left(\rho^{3} f^{\prime}(\rho)+\frac{2}{e^{2} f_{\pi}^{2}} \rho f^{\prime}(\rho) \sin ^{2} f(\rho)\right) \\
& \sim-\frac{8 \pi \lambda i}{3} A \tau_{i} A^{\dagger} .
\end{aligned}
$$

In the last line we have used that $\rho$ is so large that we can use the asymptotic expression (3.2) for $f$ and have omitted non-leading terms. Combining this with

$$
\begin{aligned}
\int_{B_{\rho}(\boldsymbol{X})} d^{3} x l_{i}^{(1)}(\boldsymbol{x}) & =-i \lambda A \tau_{m} A^{\dagger} \int_{B_{\rho}(\boldsymbol{X})} d^{3} x \partial_{i} \partial_{m} \frac{1}{|\boldsymbol{x}-\boldsymbol{X}|} \\
& =-i \lambda A \tau_{m} A^{\dagger} \frac{1}{3} \delta_{i m} \int_{B_{\rho}(0)} d^{3} x \Delta \frac{1}{|\boldsymbol{x}|} \\
& =\frac{4 \pi \lambda i}{3} A \tau_{i} A^{\dagger}
\end{aligned}
$$

we have

$$
\int_{B_{\rho}(\boldsymbol{X})} d^{3} x\left(\bar{L}_{i}^{(1)}-l_{i}^{(1)}\right) \sim-4 \pi \lambda i A \tau_{i} A^{\dagger}
$$

Since

$$
r_{i}^{(2)}(\boldsymbol{X})=-i \lambda \frac{\delta_{i n}-3 \hat{R}_{i} \hat{R}_{n}}{R^{3}} B \tau_{n} B^{\dagger}
$$

we therefore find

$$
\operatorname{tr}\left(r_{i}^{(2)}(\boldsymbol{X}) \int_{B_{\rho}(\boldsymbol{X})} d^{3} x\left(\bar{L}_{i}^{(1)}-l_{i}^{(1)}\right)\right) \sim-8 \pi \lambda^{2} \frac{\operatorname{tr} \mathcal{O}-3 \hat{\boldsymbol{R}} \cdot \mathcal{O} \hat{\boldsymbol{R}}}{R^{3}} .
$$

An analogous calculation for the integral over $B_{\rho}(\boldsymbol{Y})$ yields also

$$
\operatorname{tr}\left(l_{i}^{(1)}(\boldsymbol{Y}) \int_{B_{\rho}(\boldsymbol{Y})} d^{3} x\left(\bar{R}_{i}^{(2)}-r_{i}^{(2)}\right)\right) \sim-8 \pi \lambda^{2} \frac{\operatorname{tr} \mathcal{O}-3 \hat{\boldsymbol{R}} \cdot \mathcal{O} \hat{\boldsymbol{R}}}{R^{3}} .
$$

To calculate the remaining integral we integrate by parts and use again $\Delta\left(|\boldsymbol{x}|^{-1}\right)=-4 \pi \delta^{(3)}(\boldsymbol{x})$ :

$$
\begin{aligned}
\int_{\mathbf{R}^{3}} d^{3} x \operatorname{tr}\left(r_{i}^{(2)} l_{i}^{(1)}\right) & =2 \lambda^{2} \mathcal{O}^{j k} \int_{\mathbf{R}^{3}} d^{3} x \partial_{i} \partial_{j} \frac{1}{|\boldsymbol{x}-\boldsymbol{X}|} \partial_{i} \partial_{k} \frac{1}{|\boldsymbol{x}-\boldsymbol{Y}|} \\
& =8 \pi \lambda^{2} \frac{\operatorname{tr} \mathcal{O}-3 \hat{\boldsymbol{R}} \cdot \mathcal{O} \hat{\boldsymbol{R}}}{R^{3}}
\end{aligned}
$$


Combining terms and introducing the constant

$$
\kappa=2 \pi \lambda^{2} f_{\pi}^{2}
$$

we obtain the result:

$$
V_{2} \sim-2 \kappa \frac{\operatorname{tr} \mathcal{O}-3(\hat{\boldsymbol{R}} \cdot \mathcal{O} \hat{\boldsymbol{R}})}{R^{3}}=2 \kappa(\nabla \cdot \mathcal{O} \nabla) \frac{1}{R}
$$

Here $\nabla$ is the gradient operator with respect to $\boldsymbol{R}$. In the following we will use the symbols $\partial_{i}$ and $\nabla$ for differentiation with respect to either $\boldsymbol{x}$ or $\boldsymbol{R}$. Which of the two possibilities applies will be clear from the context. Note that one would get $-V_{2}$ from integrating the asymptotic expression $\operatorname{tr}\left(l_{i}^{(1)} r_{i}^{(2)}\right)$ over the whole of $\mathbf{R}^{3}$. The effect of replacing the singular part of $l_{i}^{(1)}$ and $r_{i}^{(2)}$ by the true, smooth solutions inside the balls is to add $2 \times V_{2}$.

One can usefully write $V_{2}$ by expressing the rotation matrix $\mathcal{O}$ in terms of its axis $\boldsymbol{n}$ and the angle of rotation about that axis $\psi$, i.e.

$$
\mathcal{O}_{i j}=\cos \psi \delta_{i j}+(1-\cos \psi) n_{i} n_{j}+\sin \psi \epsilon_{i j k} n_{k}
$$

and finds

$$
V_{2} \sim-2 \kappa(1-\cos \psi) \frac{1-3(\hat{\boldsymbol{R}} \cdot \boldsymbol{n})^{2}}{R^{3}}
$$

Although the details of our calculations may seem quite complicated, the essential steps are simple. After suitably dividing the region of integration we were able to express the integral for (4.8) involving $U(\boldsymbol{x})$ at all points in $\mathbf{R}^{3}$ in the simple form (5.15) which requires only information about the asymptotic nature of the fields. This remarkable result rests essentially on the fact that the two $B=1$ Skyrmions that enter the product ansatz individually satisfy the equations of motion, as expressed in (5.3).

There is another, heuristic way of deriving (5.15). It is based on the observation, explained in sect. 3, that, from afar, a Skyrmion looks like a triplet of orthogonal scalar dipoles. Thus it is plausible that the long-range static potential between two Skyrmions can be understood in terms of scalar dipole interactions. Specifically one considers two triplets $\left\{\boldsymbol{p}_{a}\right\}$ and $\left\{\boldsymbol{q}_{a}\right\}, a=1,2,3$ of scalar dipoles. There is a scalar dipole interaction between $\boldsymbol{p}_{a}$ and $\boldsymbol{q}_{b}$ only if $a=b$, given by

$$
\frac{1}{4 \pi}\left(\boldsymbol{p}_{a} \cdot \nabla\right)\left(\boldsymbol{q}_{a} \cdot \nabla\right) \frac{1}{R}
$$

To obtain the total potential we simply sum the interactions of the pairs. We also need the formula for the dipole moments associated to a Skyrmion in general orientation (3.7). Let $\mathcal{A}$ and $\mathcal{B}$ be the $S O(3)$ matrices associated to the $S U(2)$ matrices $A$ and $B$ as in (2.13). Then we have from (3.7)

$$
\boldsymbol{p}_{a}=4 \pi f_{\pi} \lambda \mathcal{A}^{-1} \boldsymbol{e}_{a} \quad \text { and } \quad \boldsymbol{q}_{a}=4 \pi f_{\pi} \lambda \mathcal{B}^{-1} \boldsymbol{e}_{a}
$$


so that $\boldsymbol{p}_{a}=\mathcal{O} \boldsymbol{q}_{a}$. Thus the potential for the interaction of two triplets of scalar dipoles is

$$
\tilde{V}_{2}=2 \kappa(\nabla \cdot \mathcal{O} \nabla) \frac{1}{R}
$$

which agrees with the asymptotic form of $V_{2}$.

\section{Skyrmions as Triplets of Scalar Dipoles}

In this section we take the dipole model further: we calculate the velocity-dependent forces between two triplets of scalar dipoles.

How do moving scalar dipoles interact? To answer this question we need to know the coupling between a massless scalar field and a general source and to write down the charge distribution for a moving and spinning scalar dipole. We fix the coupling of a scalar field $\phi$ to a general charge distribution $\rho$ by writing the field Lagrangian density as

$$
\mathcal{L}_{\text {field }}=\frac{1}{2} \partial_{\mu} \phi \partial^{\mu} \phi+\rho \phi
$$

For given $\rho$ this leads to the wave equation

$$
\frac{\partial^{2} \phi}{\partial t^{2}}-\Delta \phi=\rho
$$

which is solved by

$$
\phi(t, \boldsymbol{x})=\frac{1}{4 \pi} \int d^{3} x^{\prime} \frac{\rho\left(t-\left|\boldsymbol{x}-\boldsymbol{x}^{\prime}\right|, \boldsymbol{x}^{\prime}\right)}{\left|\boldsymbol{x}-\boldsymbol{x}^{\prime}\right|} .
$$

Turning to the charge distribution $\rho$ we notice that it must be a Lorentz scalar to make equation (6.2) consistent. As a first step we give the charge distribution of a moving point particle. In its rest frame is is characterised by a coupling strength $g$ and its position $\boldsymbol{X}$ and is given by

$$
g \delta^{(3)}(\boldsymbol{x}-\boldsymbol{X})
$$

To find its charge distribution in a general frame we write down a charge distribution which is manifestly a Lorentz scalar and which reduces to (6.4) in the particle's rest frame. For a particle with world line $X(\tau)=(T(\tau), \boldsymbol{X}(\tau))$ and four-velocity $u=\left(u^{0}, \boldsymbol{u}\right)=d X / d \tau$ the required distribution is

$$
\rho_{\text {point }}(x)=g \int d \tau \sqrt{u_{\mu} u^{\mu}} \delta^{(4)}(x-X(\tau)) .
$$

Carrying out the integration over the world line parameter $\tau$ and using

$$
\left(\frac{d T}{d \tau}\right)^{-1} \sqrt{u_{\mu} u^{\mu}}=\sqrt{1-\boldsymbol{u}^{2}}
$$


we find

$$
\rho_{\text {point }}(t, \boldsymbol{x})=g \sqrt{1-\boldsymbol{u}^{2}} \delta^{(3)}(\boldsymbol{x}-\boldsymbol{X}(t))
$$

where, in an abuse of notation, we have written $\boldsymbol{X}(t)$ for $\boldsymbol{X}\left(T^{-1}(t)\right)$.

Our model for a scalar dipole involves a world line $X(\tau)$ and the dipole moment $\boldsymbol{p}(\tau)$ in the dipole's rest frame. In that frame, which is characterised by $u=d X / d \tau=(1,0)$, we can think of the dipole as consisting of two equal and opposite scalar point charges with rest charges $\pm g$, situated at $\boldsymbol{X}+\frac{1}{2} \boldsymbol{d}$ and $\boldsymbol{X}-\frac{1}{2} \boldsymbol{d}$, see fig. 1 .

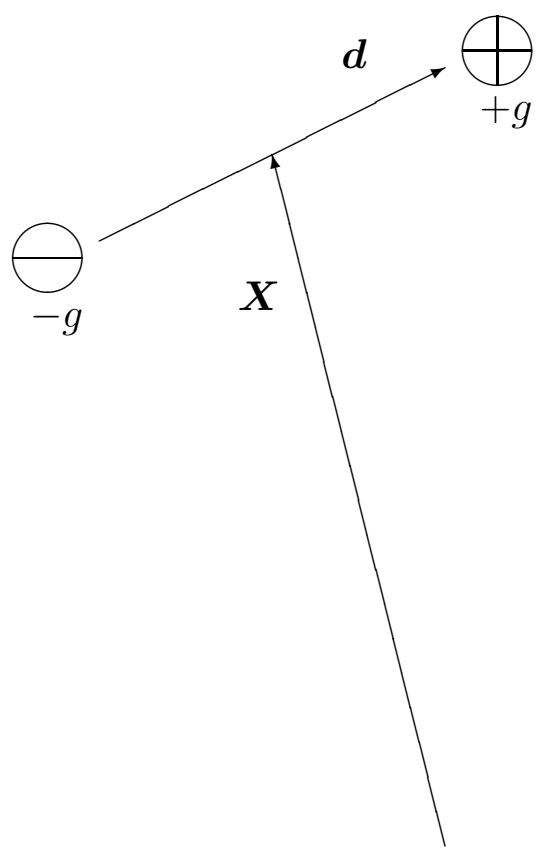

Fig. 1

Dipole charge distribution in the dipole's rest frame

Still in the rest frame we impose that $|\boldsymbol{d}|$ remains constant during time evolution and introduce the angular velocity $\boldsymbol{\alpha}$

$$
\frac{d \boldsymbol{d}}{d \tau}=\boldsymbol{d} \times \boldsymbol{\alpha}
$$


Then the charge distribution depicted in fig. 1 is described by

$$
g \sqrt{1-\left(\frac{d \boldsymbol{d}}{d \tau}\right)^{2}} \delta^{(3)}\left(\boldsymbol{x}-\left(\boldsymbol{X}+\frac{1}{2} \boldsymbol{d}\right)\right)-g \sqrt{1-\left(\frac{d \boldsymbol{d}}{d \tau}\right)^{2}} \delta^{(3)}\left(\boldsymbol{x}-\left(\boldsymbol{X}-\frac{1}{2} \boldsymbol{d}\right)\right) .
$$

In the limit where $|\boldsymbol{d}|$ becomes small and $g$ large in such a way that the dipole moment

$$
\boldsymbol{p}=g \boldsymbol{d}
$$

remains constant this simplifies to

$$
-\boldsymbol{p} \cdot \nabla \delta^{(3)}(\boldsymbol{x}-\boldsymbol{X})
$$

Again we write this charge distribution in a general frame by giving a Lorentz scalar distribution which reduces to 6.11 in the rest frame $u=(1,0)$ :

$$
\rho_{\text {dipole }}(x)=-\int d \tau \sqrt{u_{\mu} u^{\mu}} P_{\mu}(\tau) \partial^{\mu} \delta^{(4)}(x-X(\tau))
$$

Here the four-vector $P$ reduces to the dipole moment $\boldsymbol{p}$ in the dipole's rest frame, so in particular $u_{\mu} P^{\mu}=0$. This condition determines $P$ in terms of $\boldsymbol{p}$ :

$$
\begin{aligned}
P^{0} & =\gamma(\boldsymbol{u} \cdot \boldsymbol{p}) \\
\boldsymbol{P} & =\boldsymbol{p}+\frac{\gamma^{2}}{\gamma+1}(\boldsymbol{u} \cdot \boldsymbol{p}) \boldsymbol{u}
\end{aligned}
$$

where $\gamma=\left(1-\boldsymbol{u}^{2}\right)^{-1 / 2}$ as before. Making these replacements in (6.12) and carrying out the integration over the world line parameter $\tau$ we arrive at the expression

$$
\rho_{\text {dipole }}(t, \boldsymbol{x})=-\left[\frac{d}{d t}(\boldsymbol{u} \cdot \boldsymbol{p})+\frac{1}{\gamma} \boldsymbol{p} \cdot \nabla+\left(\frac{\gamma}{\gamma+1}-\frac{1}{\gamma}\right)(\boldsymbol{u} \cdot \boldsymbol{p})(\boldsymbol{u} \cdot \nabla)\right] \delta^{3}(\boldsymbol{x}-\boldsymbol{X}(t)) .
$$

Here $\boldsymbol{p}=\boldsymbol{p}\left(T^{-1}(t)\right)$ should be thought of as a function of time. Assuming $d \boldsymbol{u} / d t \approx 0$ and retaining only terms which are at most quadratic in the velocities $\boldsymbol{u}$ and $\boldsymbol{\alpha}$ this reduces to the distribution

$$
\rho_{1}(t, \boldsymbol{x})=-\left[(\boldsymbol{u} \cdot \boldsymbol{p} \times \boldsymbol{\alpha})+\left(1-\frac{1}{2} \boldsymbol{u}^{2}\right) \boldsymbol{p} \cdot \nabla-\frac{1}{2}(\boldsymbol{u} \cdot \boldsymbol{p})(\boldsymbol{u} \cdot \nabla)\right] \delta^{3}(\boldsymbol{x}-\boldsymbol{X}(t)) .
$$

We can now calculate the potential due to $\rho_{1}$ according to (6.3). Since $\rho_{1}$ varies slowly with time for slowly moving sources we can make some approximations:

$$
\phi_{1}(t, \boldsymbol{x}) \approx \frac{1}{4 \pi}\left(\int d^{3} x^{\prime} \frac{\rho_{1}\left(t, \boldsymbol{x}^{\prime}\right)}{\left|\boldsymbol{x}-\boldsymbol{x}^{\prime}\right|}-\frac{d}{d t} \int d^{3} x^{\prime} \rho_{1}\left(t, \boldsymbol{x}^{\prime}\right)+\frac{1}{2} \frac{d^{2}}{d t^{2}} \int d^{3} x^{\prime} \rho_{1}\left(t, \boldsymbol{x}^{\prime}\right)\left|\boldsymbol{x}^{\prime}-\boldsymbol{x}\right|\right)
$$

We should be careful to understand the range of validity of our approximation: for constant $\boldsymbol{p}$ but time-varying $\boldsymbol{X}$ we can neglect higher order terms in the above Taylor expansion if $\boldsymbol{u}$ is small relative to the speed of light and the acceleration and higher derivatives are small. For fixed $\boldsymbol{X}$ 
and time dependent $\boldsymbol{p}$, however, higher terms in the Taylor series are not necessarily small even if $\boldsymbol{p}$ rotates uniformly. In that case the dipole field is exactly given by (3.9) and (6.16) is an expansion in powers of $|\boldsymbol{\alpha}||\boldsymbol{x}-\boldsymbol{X}|$ of the exact solution. Retaining only terms that contain at most two time derivatives we obtain

$$
\left.\phi_{1}(t, \boldsymbol{x}) \approx \frac{1}{4 \pi}\left((\boldsymbol{u} \cdot \boldsymbol{\alpha} \times \boldsymbol{p})-\left(1-\frac{1}{2} \boldsymbol{u}^{2}\right) \boldsymbol{p} \cdot \nabla+\frac{1}{2}(\boldsymbol{u} \cdot \boldsymbol{p})(\boldsymbol{u} \cdot \nabla)\right) \frac{1}{|\boldsymbol{x}-\boldsymbol{X}|}-\frac{1}{2} \frac{d^{2}}{d t^{2}} \boldsymbol{p} \cdot \nabla|\boldsymbol{x}-\boldsymbol{X}|\right) .
$$

Now consider a second scalar dipole with world line $Y(\sigma)=(S(\sigma), \boldsymbol{Y}(\sigma))$ and with dipole moment $\boldsymbol{q}$ in its rest frame. Denote its three-velocity $d \boldsymbol{Y} / d t$ by $\boldsymbol{v}$ and its angular velocity in its rest frame by $\boldsymbol{\beta}$, i.e. $d \boldsymbol{q} / d \sigma=\boldsymbol{q} \times \boldsymbol{\beta}$. Its charge distribution, in the same approximation as for the first dipole, is

$$
\rho_{2}(t, \boldsymbol{x})=-\left[(\boldsymbol{v} \cdot \boldsymbol{q} \times \boldsymbol{\beta})+\left(1-\frac{1}{2} \boldsymbol{v}^{2}\right)(\boldsymbol{q} \cdot \nabla)-\frac{1}{2}(\boldsymbol{v} \cdot \boldsymbol{q})(\boldsymbol{v} \cdot \nabla)\right] \delta^{3}(\boldsymbol{x}-\boldsymbol{Y}(t)) .
$$

The part of the Lagrangian which describes the interaction of the second dipole with the field produced by the first is therefore

$$
\begin{aligned}
\tilde{L}_{\mathrm{int}} & =\int d^{3} x^{\prime} \rho_{2}\left(t, \boldsymbol{x}^{\prime}\right) \phi_{1}\left(t, \boldsymbol{x}^{\prime}\right) \\
& \approx \frac{1}{4 \pi}[(\nabla \cdot \boldsymbol{p})(\boldsymbol{v} \cdot \boldsymbol{\beta} \times \boldsymbol{q})-(\boldsymbol{q} \cdot \nabla)(\boldsymbol{u} \cdot \boldsymbol{\alpha} \times \boldsymbol{p}) \\
& -\left(1-\frac{1}{2} \boldsymbol{u}^{2}-\frac{1}{2} \boldsymbol{v}^{2}\right)(\boldsymbol{p} \cdot \nabla)(\boldsymbol{q} \cdot \nabla) \\
& \left.+\frac{1}{2}(\boldsymbol{u} \cdot \boldsymbol{p})(\boldsymbol{u} \cdot \nabla)(\boldsymbol{q} \cdot \nabla)+\frac{1}{2}(\boldsymbol{v} \cdot \boldsymbol{q})(\boldsymbol{v} \cdot \nabla)(\boldsymbol{p} \cdot \nabla)\right] \frac{1}{R} \\
& -\frac{1}{8 \pi}(\boldsymbol{q} \cdot \nabla) \frac{d^{2}}{d t^{2}}(\boldsymbol{p} \cdot \nabla) R .
\end{aligned}
$$

As in the previous section $\boldsymbol{R}=\boldsymbol{X}-\boldsymbol{Y}, R=|\boldsymbol{R}|$. In the last line $\nabla$ differentiates with respect to $\boldsymbol{R}$ and $d / d t$, when operating on $R$, acts on $\boldsymbol{X}$ only. It is useful to rewrite the last term

$$
\begin{aligned}
(\boldsymbol{q} \cdot \nabla) \frac{d^{2}}{d t^{2}}(\boldsymbol{p} \cdot \nabla) R= & \frac{d}{d t}\left((\boldsymbol{q} \cdot \nabla) \frac{d}{d t}(\boldsymbol{p} \cdot \nabla) R\right) \\
& -(\dot{\boldsymbol{q}} \cdot \nabla)(\dot{\boldsymbol{p}} \cdot \nabla) R-(\dot{\boldsymbol{q}} \cdot \nabla)(\boldsymbol{p} \cdot \nabla)(\boldsymbol{u} \cdot \nabla) R \\
& +(\boldsymbol{q} \cdot \nabla)(\dot{\boldsymbol{p}} \cdot \nabla)(\boldsymbol{v} \cdot \nabla) R+(\boldsymbol{q} \cdot \nabla)(\boldsymbol{p} \cdot \nabla)(\boldsymbol{u} \cdot \nabla)(\boldsymbol{v} \cdot \nabla) R .
\end{aligned}
$$

The time derivative outside the big brackets in the first line acts on all the dynamical variables $\boldsymbol{p}, \boldsymbol{q}, \boldsymbol{X}, \boldsymbol{Y}$ so

$$
\frac{d}{d t}\left((\boldsymbol{q} \cdot \nabla) \frac{d}{d t}(\boldsymbol{p} \cdot \nabla) R\right)
$$


is a total time derivative which we may discard since we are working with a Lagrangian.

Finally we translate our results into "Skyrme language", using the notation introduced in sect. 5. Discarding the total time derivative in (6.20) and summing over three pairs of dipoles $\boldsymbol{p}_{a}$ and $\boldsymbol{q}_{a}$ we find, using the formulae (5.19),

$$
\begin{aligned}
\tilde{L}_{\text {int }} & =\kappa[2(\nabla \cdot \mathcal{O}(\boldsymbol{v} \times \boldsymbol{\beta}))-2(\boldsymbol{u} \times \boldsymbol{\alpha} \cdot \mathcal{O} \nabla) \\
& \left.+\left(\boldsymbol{u}^{2}+\boldsymbol{v}^{2}\right)(\nabla \cdot \mathcal{O} \nabla)+(\boldsymbol{u} \cdot \nabla)(\boldsymbol{u} \cdot \mathcal{O} \nabla)+(\boldsymbol{v} \cdot \nabla)(\nabla \cdot \mathcal{O} \boldsymbol{v})\right] \frac{1}{R} \\
& +\kappa[(\nabla \times \boldsymbol{\alpha} \cdot \mathcal{O}(\nabla \times \boldsymbol{\beta})) \\
& +(\boldsymbol{v} \cdot \nabla)(\nabla \times \boldsymbol{\alpha} \cdot \mathcal{O} \nabla)-(\boldsymbol{u} \cdot \nabla)(\nabla \cdot \mathcal{O}(\nabla \times \boldsymbol{\beta})) \\
& -(\nabla \cdot \mathcal{O} \nabla)(\boldsymbol{u} \cdot \nabla)(\boldsymbol{v} \cdot \nabla)] R \\
& -2 \kappa(\nabla \cdot \mathcal{O} \nabla) \frac{1}{R} .
\end{aligned}
$$

Note that the terms in this Lagrangian can be classified into three types: spin-spin couplings which couple the angular velocities $\boldsymbol{\alpha}$ and $\boldsymbol{\beta}$ to each other with strength proportional to $1 / R$, spin-orbit couplings which couple $\boldsymbol{\alpha}$ and $\boldsymbol{\beta}$ to $\boldsymbol{u}$ and $\boldsymbol{v}$ with strength proportional to $1 / R^{2}$ and orbit-orbit couplings which are quadratic in $\boldsymbol{u}$ and $\boldsymbol{v}$ and which are of order $1 / R^{3}$. Before we discuss our result further we derive it from the relativised product ansatz.

\section{Two-Skyrmion Dynamics according to the Relativised Product Ansatz}

In this section we evaluate the interaction Lagrangian $L_{\text {int }}$ on the time-dependent relativised product ansatz up to and including terms which are quadratic in the velocities $\boldsymbol{u}, \boldsymbol{v}, \boldsymbol{\alpha}$ and $\boldsymbol{\beta}$. For the sake of the impatient reader, who may wish to proceed immediately to the comments at the end of this section, we announce already at this point that the asymptotic form of our final result agrees, up to a total time derivative, with the interaction Lagrangian $\tilde{L}_{\text {int }}$ calculated in the previous section.

We begin with the following kinetic terms of the interaction Lagrangian:

$$
T_{\text {int }}=\frac{f_{\pi}^{2}}{2} \int d^{3} x \operatorname{tr}\left(L_{0}^{(1)} \bar{R}_{0}^{(2)}+\bar{L}_{0}^{(1)} R_{0}^{(2)}-L_{0}^{(1)} R_{0}^{(2)}\right) .
$$

For time dependent fields, $L_{\text {int }}$ actually contains further terms with explicit time derivatives in the double commutator $\left[L_{0}^{(1)},\left[L_{0}^{(1)}, L_{i}^{(1)}\right]\right]$ contained in $\bar{L}_{i}^{(1)}$ (similarly for $\bar{R}_{i}^{(2)}$ ) but for computational purposes it is best initially to concentrate on $T_{\text {int }}$. Since each term in $T_{\text {int }}$ explicitly contains two time derivatives we can omit the relativistic corrections in the product ansatz (4.1) when calculating (7.1) since we only retain terms quadratic in the velocities anyway. Thus we replace 
$t^{\prime}$ and $t^{\prime \prime}$ by $\delta t, \boldsymbol{\xi}$ by $\boldsymbol{x}-\boldsymbol{X}-\boldsymbol{u} \delta t$ and $\boldsymbol{\eta}$ by $\boldsymbol{x}-\boldsymbol{Y}-\boldsymbol{v} \delta t$ in (4.1). To carry out the integration in (7.1) we employ the same technique as for the static terms, so we divide $\mathbf{R}^{3}$ as in (4.9), extend the integration for the asymptotic expressions of the fields from $V_{\rho}(\boldsymbol{X}, \boldsymbol{Y})$ to the whole of $\mathbf{R}^{3}$, subtract the integrals of the singular parts over the balls and add the integrals of the true fields over the balls. This time we will omit most of the details, but it is instructive to write down the integrals of the asymptotic expressions explicitly. These now read

$$
l_{0}^{(1)}(t, \boldsymbol{x})=i \lambda A\left(\epsilon_{i j k} \alpha_{i} \tau_{j} \partial_{k} \frac{1}{|\boldsymbol{x}-\boldsymbol{X}|}+u_{i} \tau_{j} \partial_{i} \partial_{j} \frac{1}{|\boldsymbol{x}-\boldsymbol{X}|}\right) A^{\dagger}
$$

and

$$
r_{0}^{(2)}(t, \boldsymbol{x})=-i \lambda B\left(\epsilon_{i j k} \beta_{i} \tau_{j} \partial_{k} \frac{1}{|\boldsymbol{x}-\boldsymbol{Y}|}+v_{i} \tau_{j} \partial_{i} \partial_{j} \frac{1}{|\boldsymbol{x}-\boldsymbol{Y}|}\right) B^{\dagger}
$$

so that

$$
\begin{aligned}
\frac{f_{\pi}^{2}}{2} \int_{\mathbf{R}^{3}} d^{3} x \operatorname{tr}\left(l_{0}^{(1)} r_{0}^{(2)}\right) & =f_{\pi}^{2} \lambda^{2}\left[\epsilon_{i j k} \epsilon_{l m n} \mathcal{O}_{j m} \alpha_{i} \beta_{l} \int d^{3} x \partial_{k} \frac{1}{|\boldsymbol{x}-\boldsymbol{X}|} \partial_{n} \frac{1}{|\boldsymbol{x}-\boldsymbol{Y}|}\right. \\
& +\epsilon_{i j k} \alpha_{i} v_{l} \mathcal{O}_{j m} \int d^{3} x \partial_{k} \frac{1}{|\boldsymbol{x}-\boldsymbol{X}|} \partial_{l} \partial_{m} \frac{1}{|\boldsymbol{x}-\boldsymbol{Y}|} \\
& +\epsilon_{l m n} \beta_{l} u_{i} \mathcal{O}_{j m} \int d^{3} x \partial_{i} \partial_{j} \frac{1}{|\boldsymbol{x}-\boldsymbol{X}|} \partial_{n} \frac{1}{|\boldsymbol{x}-\boldsymbol{Y}|} \\
& \left.+u_{i} v_{l} \mathcal{O}_{j m} \int d^{3} x \partial_{i} \partial_{j} \frac{1}{|\boldsymbol{x}-\boldsymbol{X}|} \partial_{l} \partial_{m} \frac{1}{|\boldsymbol{x}-\boldsymbol{Y}|}\right]
\end{aligned}
$$

We use the first of the above integrals to illustrate our method of integration: integrating by parts, shifting the origin to $\boldsymbol{Y}$ we get

$$
\begin{aligned}
\int d^{3} x \partial_{k} \frac{1}{|\boldsymbol{x}-\boldsymbol{X}|} \partial_{n} \frac{1}{|\boldsymbol{x}-\boldsymbol{Y}|} & =-\int d^{3} x \frac{1}{|\boldsymbol{x}|} \partial_{n} \partial_{k} \frac{1}{|\boldsymbol{x}-\boldsymbol{R}|} \\
& =-\int d^{3} x \frac{1}{|\boldsymbol{x}|} \frac{\partial}{\partial R_{n}} \frac{\partial}{\partial R_{k}} \frac{1}{|\boldsymbol{x}-\boldsymbol{R}|}
\end{aligned}
$$

Next we would like to take the differentiation outside the integral. To justify this interchange of limits we consider the above integrals over a large, but finite ball $B_{K}(0)$ with radius $K>R$, centered at the origin. Then

$$
\int_{B_{K}(0)} d^{3} x \frac{1}{|\boldsymbol{x}-\boldsymbol{R}|} \frac{1}{|\boldsymbol{x}|}=4 \pi K-2 \pi R
$$

For the finite region of integration, the exchange of limits is allowed, so

$$
\begin{aligned}
\int_{B_{K}(0)} d^{3} x \frac{1}{|\boldsymbol{x}|} \frac{\partial}{\partial R_{n}} \frac{\partial}{\partial R_{k}} \frac{1}{|\boldsymbol{x}-\boldsymbol{R}|} & =\frac{\partial}{\partial R_{n}} \frac{\partial}{\partial R_{k}}(4 \pi \kappa-2 \pi R) \\
& =-2 \pi \partial_{k} \partial_{n} R
\end{aligned}
$$


We see that, as long as $K>R$, the integral on the left is actually independent of $K$, we can take the limit $K \rightarrow \infty$ and recover the original integral. Evaluating the other integrals in (7.4) in a similar fashion we obtain

$$
\begin{aligned}
\frac{f_{\pi}^{2}}{2} \int_{\mathbf{R}^{3}} d^{3} x \operatorname{tr}\left(l_{0}^{(1)} r_{0}^{(2)}\right) & =\kappa[(\nabla \times \boldsymbol{\alpha} \cdot \mathcal{O}(\nabla \times \boldsymbol{\beta})) R \\
& +(\boldsymbol{v} \cdot \nabla)(\nabla \times \boldsymbol{\alpha} \cdot \mathcal{O} \nabla) R \\
& -(\boldsymbol{u} \cdot \nabla)(\nabla \cdot \mathcal{O}(\nabla \times \boldsymbol{\beta})) R \\
& -(\nabla \cdot \mathcal{O} \nabla)(\boldsymbol{u} \cdot \nabla)(\boldsymbol{v} \cdot \nabla) R]
\end{aligned}
$$

How is this result affected when we take into account the true form of $\bar{L}_{0}^{(1)}$ and $\bar{R}_{0}^{(2)}$ ? As in the static case, this amounts to adding

$$
\frac{f_{\pi}}{2} \operatorname{tr}\left(r_{0}^{(2)}(\boldsymbol{X}) \int_{B_{\rho}(\boldsymbol{X})} d^{3} x\left(\bar{L}_{0}^{(1)}-l_{0}^{(1)}\right)\right)
$$

and

$$
\frac{f_{\pi}}{2} \operatorname{tr}\left(l_{0}^{(1)}(\boldsymbol{Y}) \int_{B_{\rho}(\boldsymbol{Y})} d^{3} x\left(\bar{R}_{0}^{(2)}-r_{0}^{(2)}\right)\right) .
$$

The net effect of this is to change the signs of some but not all of the terms generated when the differentiation in $(\overline{7.8})$ is carried out. The terms in the integrand of (7.9) which are proportional to $\boldsymbol{\alpha}$ and the terms in the integrand of (7.10) which are proportional to $\boldsymbol{\beta}$ lead to spin-spin terms which fall off like $1 / R^{2}$ and to spin-orbit terms which fall off like $1 / R^{3}$. Such terms are non-leading and we neglect them. Thus for our purposes $\bar{L}_{0}^{(1)}=-u_{i} \bar{L}_{i}^{(1)}$ and $\bar{R}_{0}^{(2)}=-v_{i} \bar{R}_{i}^{(2)}$. Hence we can again exploit the fact that the vector fields $\overline{\boldsymbol{L}}^{(1)}$ and $\overline{\boldsymbol{R}}^{(2)}$ are divergenceless, but we do not give the details here. The result is

$$
\begin{aligned}
T_{\mathrm{int}} & \sim \kappa[(\nabla \times \boldsymbol{\alpha} \cdot \mathcal{O}(\nabla \times \boldsymbol{\beta})) R \\
& +(\boldsymbol{v} \cdot \nabla)(\nabla \times \boldsymbol{\alpha} \cdot \mathcal{O} \nabla) R-2(\nabla \times \boldsymbol{\alpha} \cdot \mathcal{O} \boldsymbol{v}) \frac{1}{R} \\
& -(\boldsymbol{u} \cdot \nabla)(\nabla \cdot \mathcal{O}(\nabla \times \boldsymbol{\beta})) R+2(\boldsymbol{u} \cdot \mathcal{O}(\nabla \times \boldsymbol{\beta})) \frac{1}{R} \\
& \left.-(\nabla \cdot \mathcal{O} \nabla)(\boldsymbol{u} \cdot \nabla)(\boldsymbol{v} \cdot \nabla) R+2(\boldsymbol{u} \cdot \mathcal{O} \nabla)(\boldsymbol{v} \cdot \nabla) \frac{1}{R}+2(\nabla \cdot \mathcal{O} \boldsymbol{v})(\boldsymbol{u} \cdot \nabla) \frac{1}{R}\right]
\end{aligned}
$$

Next we evaluate the potential energy $V_{2}$ (5.1) on the relativised product ansatz (4.1), again setting $\delta t=0$ after the calculation. The velocity dependence of that field will lead to velocitydependent modifications of the potential calculated in sect. 5. As a first step we restrict attention to constant matrix functions $A\left(t^{\prime}\right)$ and $B\left(t^{\prime \prime}\right)$, i.e. vanishing angular velocities. We then introduce 
a "rest frame current" with components $L_{\xi_{i}}^{(1)}$ via

$$
L_{\xi_{i}}^{(1)}=U^{(1) \dagger} \frac{\partial}{\partial \xi_{i}} U^{(1)}
$$

and find

$$
L_{i}^{(1)}=L_{\xi_{i}}^{(1)}+\frac{1}{2} u_{i}\left(u_{j} L_{\xi_{j}}^{(1)}\right)
$$

Further we define the current with components

$$
\bar{L}_{\xi_{i}}^{(1)}=L_{\xi_{i}}^{(1)}-\frac{1}{4 e^{2} f_{\pi}^{2}}\left[L_{\xi_{j}}^{(1)},\left[L_{\xi_{j}}^{(1)}, L_{\xi_{i}}^{(1)}\right]\right]
$$

which satisfies

$$
\frac{\partial}{\partial \xi_{i}} \bar{L}_{\xi_{i}}^{(1)}=0
$$

Carefully taking into account the explicit time derivatives in the double commutator $\left[L_{0}^{(1)},\left[L_{0}^{(1)}, L_{i}^{(1)}\right]\right]$ contained in $\bar{L}_{i}^{(1)}$ one checks that

$$
\bar{L}_{i}^{(1)}=\bar{L}_{\xi_{i}}^{(1)}+\frac{1}{2} u_{i}\left(u_{j} \bar{L}_{\xi_{j}}^{(1)}\right)
$$

Defining

$$
R_{\eta_{i}}^{(2)}=U^{(2)} \frac{\partial}{\partial \eta_{i}} U^{(2) \dagger}
$$

and $\bar{R}_{\eta_{i}}^{(2)}$ analogously to $\bar{L}_{\xi_{i}}^{(1)}$ we also have

$$
\bar{R}_{i}^{(2)}=\bar{R}_{\eta_{i}}^{(2)}+\frac{1}{2} v_{i}\left(v_{j} \bar{R}_{\eta_{j}}^{(2)}\right)
$$

Then, when integrating $\bar{L}_{i}^{(1)}(t, \boldsymbol{x})$ over $B_{\rho}(\boldsymbol{X})$, we express $\bar{L}_{i}^{(1)}$ in terms of $\bar{L}_{\xi_{i}}^{(1)}$ according to (7.13) and change integration variables via $d^{3} x=\left(1-\frac{1}{2} \boldsymbol{u}^{2}\right) d^{3} \xi$. Thus, to order $\boldsymbol{u}^{2}$,

$$
\begin{aligned}
\int_{B_{\rho}(\boldsymbol{X})} d^{3} x \bar{L}_{i}^{(1)}(t, \boldsymbol{x}) & =\left(\left(1-\frac{1}{2} \boldsymbol{u}^{2}\right) \delta_{i m}+\frac{1}{2} u_{i} u_{m}\right) \int_{B_{\rho}(0)} d^{3} \xi \bar{L}_{\xi_{m}}^{(1)}(t, \boldsymbol{\xi}) \\
& =-\left(\left(1-\frac{1}{2} \boldsymbol{u}^{2}\right) \delta_{i m}+\frac{1}{2} u_{i} u_{m}\right) \frac{8 \pi \lambda i}{3} A \tau_{m} A^{\dagger}
\end{aligned}
$$

where we used (5.7) in the last line. The integral of $\bar{R}_{i}^{(2)}(t, \boldsymbol{x})$ over $B_{\rho}(\boldsymbol{Y})$ can be dealt with in an analogous fashion. To calculate the remaining integrals in $V_{2}$ we need expressions for the asymptotic form of $L_{i}^{(1)}$ and $R_{i}^{(2)}$ :

$l_{i}^{(1)}(t, \boldsymbol{x})=-i \lambda A \tau_{m} A^{\dagger}\left[\left(\left(1-\frac{1}{2} \boldsymbol{u}^{2}\right) \partial_{i} \partial_{m}-\frac{1}{2}(\boldsymbol{u} \cdot \nabla) u_{m} \partial_{i}\right) \frac{1}{|\boldsymbol{x}-\boldsymbol{X}|}+\frac{1}{2}(\boldsymbol{u} \cdot \nabla)(\boldsymbol{u} \cdot \nabla) \partial_{i} \partial_{m}|\boldsymbol{x}-\boldsymbol{X}|\right]$ 
and

$$
r_{i}^{(2)}(t, \boldsymbol{x})=i \lambda B \tau_{m} B^{\dagger}\left[\left(\left(1-\frac{1}{2} \boldsymbol{v}^{2}\right) \partial_{i} \partial_{m}-\frac{1}{2}(\boldsymbol{v} \cdot \nabla) v_{m} \partial_{i}\right) \frac{1}{|\boldsymbol{x}-\boldsymbol{Y}|}+\frac{1}{2}(\boldsymbol{v} \cdot \nabla)(\boldsymbol{v} \cdot \nabla) \partial_{i} \partial_{m}|\boldsymbol{x}-\boldsymbol{Y}|\right]
$$

Using these formulae it is not difficult to reduce the integrations of $l_{i}^{(1)}, r_{i}^{(2)}$ and $\operatorname{tr}\left(l_{i}^{(1)} r_{i}^{(2)}\right)$ to integrals of the same form as those appearing in $T_{\mathrm{int}}$ earlier in his section. The resulting asymptotic expression for $V_{2}$ is

$$
V_{2} \sim 2 \kappa\left[\left(1-\frac{1}{2} \boldsymbol{u}^{2}-\frac{1}{2} \boldsymbol{v}^{2}\right)(\nabla \cdot \mathcal{O} \nabla)+\frac{1}{2}(\boldsymbol{u} \cdot \nabla)(\boldsymbol{u} \cdot \mathcal{O} \nabla)+\frac{1}{2}(\boldsymbol{v} \cdot \nabla)(\nabla \cdot \mathcal{O} \boldsymbol{v})\right] \frac{1}{R} .
$$

We still have to allow for $A\left(t^{\prime}\right)$ and $B\left(t^{\prime \prime}\right)$ to vary with $t^{\prime}$ and $t^{\prime \prime}$ respectively, which leads to both being a function of $\boldsymbol{x}$. This produces an additional term in the formula $(7.20)$ for $l_{i}^{(1)}$ :

$$
i \lambda A \partial_{i}\left(\boldsymbol{u} \cdot(\boldsymbol{x}-\boldsymbol{X})(\boldsymbol{\alpha} \times \nabla \cdot \boldsymbol{\tau}) \frac{1}{|\boldsymbol{x}-\boldsymbol{X}|}\right) A^{\dagger}
$$

and a similar extra term in the formula (7.21) for $r_{i}^{(2)}$ :

$$
-i \lambda B \partial_{i}\left(\boldsymbol{v} \cdot(\boldsymbol{x}-\boldsymbol{Y})(\boldsymbol{\beta} \times \nabla \cdot \boldsymbol{\tau}) \frac{1}{|\boldsymbol{x}-\boldsymbol{Y}|}\right) B^{\dagger}
$$

Inserting these extra terms into (5.1) one thus expects to obtain extra spin-orbit terms of order $1 / R^{2}$. Further spin-orbit terms are generated if one considers the effect of the relativistic time transformation on $\bar{L}_{i}^{(1)}$ and $\bar{R}_{i}^{(2)}$, but these are of order $1 / R^{3}$ and non-leading. Miraculously, however, the sum of the extra spin-orbit terms generated when the above corrections to $l_{i}^{(1)}$ and $r_{i}^{(2)}$ are taken into account vanishes. Since all the integrals required to prove this result have appeared earlier in this section we will not go through the details here. Physically this result means that the relativity of time does not affect the long-range interaction between two Skyrmions to the accuracy considered here: we could have replaced $t^{\prime}$ and $t^{\prime \prime}$ by $\delta t$ straight away.

Before we combine $T_{\text {int }}$ and $V_{2}$ into our final expression for $L_{\text {int }}$ we exploit the fact that we can discard total derivatives in a Lagrangian. Noting that, for any two vectors $\boldsymbol{r}$ and $\boldsymbol{s}$

$$
(\boldsymbol{r} \cdot \dot{\mathcal{O}} \boldsymbol{s})=(\boldsymbol{\alpha} \times \boldsymbol{r} \cdot \mathcal{O} \boldsymbol{s})+(\boldsymbol{r} \cdot \mathcal{O}(\boldsymbol{\beta} \times \boldsymbol{s}))
$$

and neglecting terms proportional to $\ddot{\boldsymbol{u}}$ (which are of order $1 / R^{4}$ ) we find that the term

$$
[(\boldsymbol{u} \cdot \mathcal{O}(\nabla \times \boldsymbol{\beta}))+(\boldsymbol{u} \cdot \mathcal{O} \nabla)(\boldsymbol{v} \cdot \nabla)] \frac{1}{R}
$$

which occurs in $T_{\text {int }}$, can be replaced by the term

$$
[(\boldsymbol{\alpha} \times \boldsymbol{u} \cdot \mathcal{O} \nabla)+(\boldsymbol{u} \cdot \mathcal{O} \nabla)(\boldsymbol{u} \cdot \nabla)] \frac{1}{R}
$$


because the two terms differ only by the total derivative $\frac{d}{d t}\left((\boldsymbol{u} \cdot \mathcal{O} \nabla) R^{-1}\right)$. Similarly we can replace

$$
[-(\nabla \times \boldsymbol{\alpha} \cdot \mathcal{O} \boldsymbol{v})+(\nabla \cdot \mathcal{O} \boldsymbol{v})(\boldsymbol{u} \cdot \nabla)] \frac{1}{R}
$$

by

$$
[(\nabla \cdot \mathcal{O}(\boldsymbol{v} \times \boldsymbol{\beta}))+(\nabla \cdot \mathcal{O} \boldsymbol{v})(\boldsymbol{v} \cdot \nabla)] \frac{1}{R}
$$

since the two terms differ by $\frac{d}{d t}\left((\nabla \cdot \mathcal{O} \boldsymbol{v}) R^{-1}\right)$.

With these replacements we have finally shown that the asymptotic form of $L_{\text {int }}$ is

$$
L_{\text {int }} \sim \tilde{L}_{\text {int }} .
$$

We already classified the terms in $\tilde{L}_{\text {int }}$ at the end of the previous section, but the result (7.30) deserves some further comments. First note that the Lagrangian

$$
\tilde{L}_{2}=-2 M+\frac{1}{2} M \boldsymbol{u}^{2}+\frac{1}{2} M \boldsymbol{v}^{2}+\frac{1}{2} \Lambda \boldsymbol{\alpha}^{2}+\frac{1}{2} \Lambda \boldsymbol{\beta}^{2}+\tilde{L}_{\text {int }}
$$

is not the asymptotic Lagrangian which one would obtain in the usual moduli space approximation: we have evaluated the Skyrme Lagrangian $L$ (2.17) on a set of velocity-dependent fields which, at a given time, differ from fields in the moduli space $\mathcal{M}^{12}$ by terms of order $\boldsymbol{u}^{2}$ and $\boldsymbol{v}^{2}$. As a result the velocity-dependent terms in $\tilde{L}_{2}$ are not simply the restriction of the kinetic energy $T$ to the submanifold $\mathcal{M}^{12}$ of $\mathcal{C}$ but also include contributions from the potential energy evaluated on the relativised, and hence velocity-dependent product ansatz.

Our second comment concerns the validity of $\tilde{L}_{2}$ as an approximate Lagrangian for twoSkyrmion dynamics. This is restricted in two ways. On the one hand it is well-known that the product ansatz is only a good description of two well-separated Skyrmions. To qualify "wellseparated" we note that the numerical work in ref. [15] indicates that the product ansatz is already a good approximation for Skyrmion separations of $R \geq 1 / 2 f_{\pi} \approx 1.5$ fermi. On the other hand the allowed range of $R$ is restricted from above for spinning Skyrmions: for given individual angular velocities $\boldsymbol{\alpha}$ and $\boldsymbol{\beta}$ the field of one Skyrmion at the position of the other is only well-described by the rigidly rotating field implicit in the product ansatz if

$$
|\boldsymbol{\alpha}| R<1 \quad \text { and } \quad|\boldsymbol{\beta}| R<1 .
$$

This assumption was made explicitly in our dipole calculation. When the individual Skyrmions are spinning at the nucleon frequency $\alpha_{N}(3.15)$ the condition (7.32) becomes $R \leq 2$ fermi. In terms of the standard classification of nuclear forces according to their range 16 this restriction means that the product ansatz, even in its relativised form, can only be used to calculate the Skyrme model's predictions for intermediate-range nuclear forces. This problem is routinely ignored in the literature; we will come back to it in sect. 9 . 
Recall, finally, that effects due to the relativity of time turned out to be irrelevant, at least to the accuracy considered here. This enabled us to replace the rest frame times $t^{\prime}$ and $t^{\prime \prime}$ by the laboratory time $\delta t$. Thus we can, at a given time $t$, define the relative orientation $\mathcal{O}=\mathcal{A}^{-1} \mathcal{B}$, which in turn is essential for the notion of attractive channel fields, to be considered in the next section.

\section{Dynamics in the Most Attractive Channel}

The truncated Lagrangian system with Lagrangian $\tilde{L}_{2}$ is still rather complicated. To understand it better we restrict attention to a particular set of fields and truncate the Lagrangian system further.

It is clear from (5.17) that the static two-Skyrmion potential is most attractive if one Skyrmion is rotated by $180^{\circ}$ relative to the other about an axis orthogonal to the line joining the two Skyrmions. Such fields are said to be in the most attractive channel and they can all be obtained by acting with the symmetry group $S(2.22)$ on a family of standard fields, parametrised by the separation parameter $R$. In terms of a cartesian basis $\left\{\boldsymbol{e}_{1}, \boldsymbol{e}_{2}, \boldsymbol{e}_{3}\right\}$ of physical space such a standard field consists of one standard hedgehog at $\frac{1}{2} R \boldsymbol{e}_{3}$ and another hedgehog, rotated by $180^{\circ}$ about $\boldsymbol{e}_{2}$, at $-\frac{1}{2} \operatorname{Re}_{3}$ :

$$
U_{R}(\boldsymbol{x})=U_{H}\left(\boldsymbol{x}-\frac{1}{2} \boldsymbol{R e}_{3}\right) \tau_{2} U_{H}\left(\boldsymbol{x}+\frac{1}{2} \boldsymbol{R e}_{3}\right) \tau_{2}
$$

We can write the action of the group $E_{3} \times S O(3)_{\text {isospin }} \in S$ in terms of an $S U(2)$ matrix $G$ and its associated $S O(3)$ matrix $\mathcal{G}$ for spatial rotations, a translation vector $\boldsymbol{S}$, and an $S U(2)$ matrix $C$ for isorotations. Acting with $(G, \boldsymbol{S}, C)$ on $U_{R}(\boldsymbol{x})$ we obtain

$$
C G^{\dagger} U_{H}\left(\boldsymbol{x}-\boldsymbol{S}-\frac{1}{2} R \mathcal{G} \boldsymbol{e}_{3}\right) G \tau_{2} G^{\dagger} U_{H}\left(\boldsymbol{x}-\boldsymbol{S}+\frac{1}{2} R \mathcal{G} \boldsymbol{e}_{3}\right) G \tau_{2} C^{\dagger} .
$$

Comparing this expression with our previous parametrisation of product fields we find the following relationship between the set $\{A, B, \boldsymbol{X}, \boldsymbol{Y}\}$ and the set $\{G, \boldsymbol{S}, C\}$ :

$$
\begin{array}{r}
A=C G^{\dagger} \quad B=-i C \tau_{2} G^{\dagger} \\
\boldsymbol{X}=\boldsymbol{S}+\frac{1}{2} R \mathcal{G} \boldsymbol{e}_{3} \quad \boldsymbol{Y}=\boldsymbol{S}-\frac{1}{2} R \mathcal{G} \boldsymbol{e}_{3} .
\end{array}
$$

Thus in particular $\hat{\boldsymbol{R}}=\mathcal{G} \boldsymbol{e}_{3}$. When $G, \boldsymbol{S}$ and $C$ vary with time we also need notation for the associated velocities. For spatial rotations we define the space-fixed (or right-invariant) angular velocity $\gamma$ via

$$
-\frac{i}{2} \gamma \cdot \boldsymbol{\tau}=\dot{G} G^{\dagger}
$$

as well as the body-fixed (or left-invariant) angular velocity $\boldsymbol{\omega}$

$$
-\frac{i}{2} \boldsymbol{\omega} \cdot \boldsymbol{\tau}=G^{\dagger} \dot{G}
$$


which are related via $\mathcal{G} \boldsymbol{\omega}=\boldsymbol{\gamma}$. Introducing the left-invariant angular velocity $\boldsymbol{\Omega}$ in isospace

$$
-\frac{i}{2} \Omega \cdot \tau=C^{\dagger} \dot{C}
$$

we find the following relationships:

$$
\boldsymbol{\alpha}=\mathcal{G}(\boldsymbol{\Omega}-\boldsymbol{\omega}) \quad \boldsymbol{\beta}=\mathcal{G}\left(\mathcal{D}_{2} \boldsymbol{\Omega}-\boldsymbol{\omega}\right)
$$

where $\mathcal{D}_{i}$ is the $S O(3)$ matrix for a rotation by $180^{\circ}$ about the $i$-th axis. For the linear velocities one checks that

$$
\boldsymbol{u}=\dot{\boldsymbol{S}}+\frac{1}{2}(\dot{R} \hat{\boldsymbol{R}}+\boldsymbol{\gamma} \times \boldsymbol{R}) \quad \boldsymbol{v}=\dot{\boldsymbol{S}}-\frac{1}{2}(\dot{R} \hat{\boldsymbol{R}}+\boldsymbol{\gamma} \times \boldsymbol{R}) .
$$

Using these relationships we shall now express the restriction of $\tilde{L}_{2}$ to relativised product fields in the most attractive channel in terms of $\boldsymbol{\omega}, \Omega, \dot{R}, \dot{\boldsymbol{S}}$ and $R$. This requires lengthy calculations which we will not describe in detail. Note, however, that it is convenient to use

$$
\mathcal{O}_{i j}=-\delta_{i j}+2 n_{i} n_{j}
$$

where $\boldsymbol{n}=\mathcal{G} \boldsymbol{e}_{2}$, to simplify the terms in $\tilde{L}_{2}$. One finds for example

$$
(\boldsymbol{u} \cdot \nabla)(\boldsymbol{v} \cdot \nabla)(\nabla \cdot \mathcal{O} \nabla) R=-4 \frac{(\boldsymbol{u} \cdot \boldsymbol{n})(\boldsymbol{v} \cdot \boldsymbol{n})}{R^{3}} .
$$

After simplifying $\tilde{L}_{2}$ in this fashion it is straightforward to replace the velocities $\boldsymbol{\alpha}, \boldsymbol{\beta}, \boldsymbol{u}$ and $\boldsymbol{v}$ as in (8.7) and (8.8).

Before we state the result we note some features which one can derive without any calculations. Combining the inversion map $\Pi\left(2.21\right.$ ) with rotations by $180^{\circ}$ in both space and isospace we obtain 9 isospace-space reflections

$$
\Pi_{a i}: U(\boldsymbol{x}) \mapsto \tau_{a} U^{\dagger}\left(\Pi_{i} \boldsymbol{x}\right) \tau_{a}
$$

where $\Pi_{i}$ is the reflection in the plane orthogonal to $\boldsymbol{e}_{i}$. Writing these maps more explicitly in terms of the pion fields we have for example

$$
\Pi_{13}: \boldsymbol{\pi}(\boldsymbol{x}) \mapsto\left(-\pi_{1}\left(x_{1}, x_{2},-x_{3}\right), \pi_{2}\left(x_{1}, x_{2},-x_{3}\right), \pi_{3}\left(x_{1}, x_{2},-x_{3}\right)\right) .
$$

The maps $\Pi_{a i}$ are symmetries of the Skyrme Lagrangian and, since they leave the set of product fields invariant, they are also symmetries of the truncated Lagrangian system with Lagrangian $\tilde{L}_{2}$. In order to understand the action of the maps $\Pi_{a i}$ on the attractive channel fields it is sufficient to consider a standard field $U_{R}(\boldsymbol{x})$ (8.1). Such a field is only strictly invariant under the action of $\Pi_{13}$ but it also invariant under the action of $\Pi_{11}$ and $\Pi_{22}$ modulo the commutator of two wellseparated hedgehog fields. In our calculations of the asymptotic forces such commutators are ignored. This can be seen from the fact that $\tilde{L}_{2}$ is symmetric under the exchange of the individual 
Skyrmions position, orientation and linear and angular velocities, although the product ansatz (4.1) is not invariant under these operations. It was shown by Verbaarschot et al. [14 that the true attractive channel fields in standard orientation and position, obtained by minimising the static potential energy for a fixed value of $R$, are in fact invariant under the symmetries $\Pi_{13}, \Pi_{11}$ and $\Pi_{22}$. This suggests that one should only trust the attractive channel fields of the product form in the asymptotic limit, where they also have those symmetries.

The invariance (modulo commutator) of $U_{R}(\boldsymbol{x})$ under $\Pi_{13}, \Pi_{11}$ and $\Pi_{22}$ implies that, for each $R$, these maps act on the orbit of $U_{R}(\boldsymbol{x})$ under $E_{3} \times S O(3)_{\text {isospin }}$. Explicitly we find, for $(a, i)=$ $(1,3),(1,1),(2,2)$ :

$$
\prod_{a i}: S \mapsto \prod_{i} \boldsymbol{S} \quad G \mapsto \tau_{i} G \tau_{i} \quad C \mapsto \tau_{a} G \tau_{a} .
$$

For the angular velocities this implies

$$
\Pi_{a i}: \omega \mapsto \mathcal{D}_{i} \omega \quad \Omega \mapsto \mathcal{D}_{a} \Omega .
$$

The restriction of $\tilde{L}_{2}$ to the orbit of the one-parameter family of fields $\left\{U_{R}(\boldsymbol{x})\right\}$ under $E_{3} \times$ $S O(3)_{\text {isospin }}$, which we denote by $L_{\text {att }}$, must be invariant under (8.13) and (8.14) and also under the left action of $E_{3} \times S O(3)_{\text {isospin }}$ on itself. This implies that the most general form of $L_{\text {att }}$ in the centre-of-mass frame $(\dot{\boldsymbol{S}}=0)$ is

$$
\mu \dot{R}^{2}+a^{2} \omega_{1}^{2}+d^{2} \omega_{2}^{2}+c^{2} \omega_{3}^{2}+A^{2} \Omega_{1}^{2}+B^{2} \Omega_{2}^{2}+C^{2} \Omega_{3}^{2}+2 e \omega_{2} \Omega_{2}+\frac{4 \kappa}{R^{3}}
$$

where $a, c, d, e, A, B, C$ and $\mu$ are functions of $R$ only. For later use it is best to "complete the square" and to rewrite this expression slightly:

$$
\mu \dot{R}^{2}+a^{2} \omega_{1}^{2}+b^{2} \omega_{2}^{2}+c^{2} \omega_{3}^{2}+A^{2} \Omega_{1}^{2}+B^{2}\left(\Omega_{2}+w \omega_{2}\right)^{2}+C^{2} \Omega_{3}^{2}+\frac{4 \kappa}{R^{3}}
$$

where $b^{2}=d^{2}-e^{2} / B^{2}$ and $w=e / B^{2}$.

Carrying out the explicit calculation of $L_{\text {att }}$ we find

$$
\begin{aligned}
L_{\text {att }} & =\left(M-\frac{2 \kappa}{R^{3}}\right) \dot{\boldsymbol{S}}^{2}-\frac{6 \kappa}{R^{3}}(\dot{\boldsymbol{S}} \cdot \hat{\boldsymbol{R}})^{2}+\left(\frac{M}{4}-\frac{2 \kappa}{R^{3}}\right) \dot{R}^{2} \\
& +\left(\frac{M}{4} R^{2}+\Lambda-\frac{15 \kappa}{2 R}\right) \omega_{1}^{2}+\left(\frac{M}{4} R^{2}+\Lambda-\frac{7 \kappa}{2 R}\right) \omega_{2}^{2}+\Lambda \omega_{3}^{2} \\
& +\left(\Lambda+\frac{\kappa}{R}\right) \Omega_{1}^{2}+\left(\Lambda-\frac{\kappa}{R}\right) \Omega_{2}^{2}+\Lambda \Omega_{3}^{2}-2\left(\Lambda-\frac{2 \kappa}{R}\right) \omega_{2} \Omega_{2}+\frac{4 \kappa}{R^{3}} .
\end{aligned}
$$

Note that this Lagrangian is not Galilei invariant. Although the momentum $\boldsymbol{P}_{S}$ conjugate to $\dot{\boldsymbol{S}}$ is conserved one finds that in general $\ddot{\boldsymbol{S}} \neq 0$. We can, however, set $\boldsymbol{P}_{S}=0$ and it then follows that $\dot{\boldsymbol{S}}=0 . L_{\text {att }}$ then takes the form (8.15) and we read off the coefficient functions $\mu, a^{2}, c^{2}, A^{2}, B^{2}$ and $C^{2}$ directly; after bringing it into the form (8.16) we also find

$$
b^{2}(R)=\frac{M}{4} R^{2}-\frac{\kappa}{2 R} \quad \text { and } \quad w(R)=-\left(1-\frac{\kappa}{\Lambda R}\right)
$$


with corrections of order $1 / R^{2}$.

We remark that the kinetic part of $L_{\text {att }}$ can formally be interpreted in terms of a Riemannian structure on the space of attractive channel fields. Such a geometric interpretation plays an important role in the moduli space approximation to the dynamics of Bogomol'nyi-type solitons. In that context the geometric viewpoint is emphasised because the kinetic energy of the field Lagrangian can itself be thought of in terms of a Riemannian structure on the configuration space $\mathcal{C}$. It is then consistent to interpret the kinetic energy of the truncated Lagrangian in terms of an induced Riemannian structure. Recall, however, that in our case the kinetic part of the Lagrangian $\tilde{L}_{2}$ is not just the restriction of the kinetic energy (2.18) to some submanifold of the configuration space $\mathcal{C}$. Thus the geometric viewpoint, though possibly interesting, seems less well founded in our case and we will not emphasise it in the following.

The asymptotic form of the functions $A^{2}, B^{2}, C^{2}$

$$
A^{2} \sim B^{2} \sim C^{2} \sim \Lambda
$$

can easily be interpreted in terms of the Skyrmions' moments of inertia. With our normalisation of the kinetic energy the formula (8.19) implies that the asymptotic moment of inertia in isospace is $2 \Lambda$ about any axis, which is what one expects for the simultaneous isorotation of two Skyrmions with moment of inertia $\Lambda$ each. Similarly the asymptotic behaviour

$$
a^{2} \sim b^{2} \sim \frac{M}{4} R^{2}+\Lambda, \quad c^{2} \sim \Lambda
$$

can be understood in terms of the moments of inertia for the rigid rotation of two Skyrmions a distance $R$ apart: $2 \Lambda$ is the moment of inertia about the line joining the Skyrmions and, by Steiner's theorem, $(2 M)\left(\frac{R}{2}\right)^{2}+2 \Lambda$ is the moment of inertia about either of the two perpendicular directions through the midpoint of the line joining the Skyrmions and at right angles to it.

The corrections to the asymptotic expressions (8.19) and (8.20) reflect the velocity-dependent forces between moving and spinning Skyrmions. To study their effects on two-Skyrmion dynamics we will now briefly investigate the equations of motion corresponding to $L_{\text {att }}$, using the Hamiltonian formalism. Thus we introduce body-fixed angular momenta in space and isospace

$$
\boldsymbol{m}=\frac{\partial L_{\mathrm{att}}}{\partial \boldsymbol{\omega}} \quad \boldsymbol{M}=\frac{\partial L_{\mathrm{att}}}{\partial \boldsymbol{\Omega}}
$$

which are conjugate to $\boldsymbol{\omega}$ and $\boldsymbol{\Omega}$ respectively; in components

$$
\begin{array}{r}
m_{1}=2 a^{2} \omega_{1}, \quad m_{2}=2\left(b^{2}+B^{2} w^{2}\right) \omega_{2}+2 B^{2} \Omega_{2}, \quad m_{3}=2 c^{2} \omega_{3} \\
M_{1}=2 A^{2} \Omega_{1}, \quad M_{2}=2 B^{2}\left(\Omega_{2}+w \omega_{2}\right), \quad M_{3}=2 C^{2} \Omega_{3} .
\end{array}
$$

Finally defining $P_{R}=\partial L_{\text {att }} / \partial \dot{R}$ we arrive at the Hamiltonian in the centre-of-mass frame

$$
H_{\mathrm{att}}=\frac{P_{R}^{2}}{4 \mu}+\frac{m_{1}^{2}}{4 a^{2}}+\frac{\left(m_{2}-w M_{2}\right)^{2}}{4 b^{2}}+\frac{m_{3}^{2}}{4 c^{2}}+\frac{M_{1}^{2}}{4 A^{2}}+\frac{M_{2}^{2}}{4 B^{2}}+\frac{M_{3}^{2}}{4 C^{2}}-\frac{4 \kappa}{R^{3}} .
$$


The conserved quanitites are the Hamiltonian itself, the squared spatial angular momentum

$$
\boldsymbol{m}^{2}=m_{1}^{2}+m_{2}^{2}+m_{3}^{2}
$$

and the squared isospin

$$
M^{2}=M_{1}^{2}+M_{2}^{2}+M_{3}^{2}
$$

as well as the space-fixed angular momenta in space and isospace which will, however, not concern us here. Using the basic Poisson brackets

$$
\left\{m_{i}, m_{j}\right\}=-\epsilon_{i j k} m_{k}, \quad\left\{M_{i}, M_{j}\right\}=-\epsilon_{i j k} M_{k}, \quad\left\{m_{i}, M_{j}\right\}=0
$$

it is not difficult to write down the Hamiltonian equations of motion $\dot{F}=\{H, F\}$ for any function $F$ of the coordinates and momenta. Exploiting the conservation laws one can also study certain trajectories qualitatively without integrating the equations of motion.

One can, for example, satisfy the equations of motion by setting $\dot{\boldsymbol{S}}=0, \boldsymbol{m}=\left(m_{1}^{0}, 0,0\right)$ and $\boldsymbol{M}=\left(M_{1}^{0}, 0,0\right)$, where $m_{1}^{0}$ and $M_{1}^{0}$ are two positive constants, and then determine $R(t)$ from the energy conservation law

$$
E=\left(\frac{M}{4}-\frac{2 \kappa}{R^{3}}\right) \dot{R}^{2}+\frac{\left(m_{1}^{0}\right)^{2}}{M R^{2}+4 \Lambda-30 \kappa / R}+\frac{\left(M_{1}^{0}\right)^{2}}{4 \Lambda+4 \kappa / R}-\frac{4 \kappa}{R^{3}} .
$$

Using the numerical values for $M$ and $\Lambda$ given in sect. 3 we can rewrite this equation in terms of the dimensionless quantity $\tilde{R}=f_{\pi} R$, and simplify it, assuming $\tilde{R}>1$ :

$$
\frac{4 E}{M}-\frac{\left(M_{1}^{0}\right)^{2}}{4}=\dot{R}^{2}+\frac{1}{45}\left(\frac{\left(m_{1}^{0}\right)^{2}}{\tilde{R}^{2}}-\frac{\left(M_{1}^{0}\right)^{2}}{\tilde{R}}-\frac{1.8}{\tilde{R}^{3}}\right) .
$$

One understands the time evolution of $R(t)$ qualitatively by looking at the "effective potential" in round brackets. Remarkably this potential includes an isospin-dependent attractive Coulomb potential which could lead to a bound motion. For such a motion one finds that $\tilde{R} \geq 2.3 / M_{1}^{0}$. However, the angular velocity $\Omega_{1}=M_{1}^{0} / 2 \Lambda$ leads, via (8.7), to individual angular velocities $\alpha_{1}$ and $\beta_{1}$ which are also of the order $M_{1}^{0} / 2 \Lambda$ (one checks that $\omega_{1}$ is negligible) and which, by (7.32), restrict the validity of $L_{\text {att }}$ to $\tilde{R}<0.7 / M_{1}^{0}$. Thus the bound motion predicted by equation (8.28) is always outside the range of validity of $L_{\text {att }}$.

Further studies of trajectories indicate that the result of our example calculation is generic: the velocity-dependent forces encoded in coefficient functions of $L_{\text {att }}$ give only small corrections to the equations of motion obtained from the following, simpler Lagrangian in which the velocitydependent forces are ignored.

$$
\begin{aligned}
L_{\text {stat }} & =2 M \dot{\boldsymbol{S}}^{2}+\frac{M}{4} \dot{R}^{2}+\left(\frac{M}{4} R^{2}+\Lambda\right) \omega_{1}^{2}+\left(\frac{M}{4} R^{2}+\Lambda\right) \omega_{2}^{2}+\Lambda \omega_{3}^{2} \\
& +\Lambda\left(\Omega_{1}^{2}+\Omega_{2}^{2}+\Omega_{3}^{2}\right)-2 \Lambda \omega_{2} \Omega_{2}+\frac{4 \kappa}{R^{3}}
\end{aligned}
$$


Trajectories calculated with $L_{\text {att }}$ are only qualitatively different from those calculated with $L_{\text {stat }}$ if the momenta $\boldsymbol{m}$ and $\boldsymbol{M}$ are taken to be so large that $L_{\text {att }}$ is no longer a good approximation for two-Skyrmion dynamics.

The calculations in this section and the previous one were based on moduli space ideas, with some relativistic corrections. The dipole picture suggests that this approach is fundamentally limited by the condition (7.32) and consequently only capable of calculating small velocity-dependent effects. However, the dipole model also provides an alternative method for studying the long-range forces between spinning Skyrmions which is in principle valid for any value of the Skyrmions' angular velocities and any (sufficiently large) value of $R$. This point of view is elaborated in the following section.

\section{Long-range Forces between Spinning Skyrmions and Nuclear Forces}

The semiclassical angular speed $\alpha_{N}$ (3.15) at which a Skyrmion has to rotate to have a nucleon's spin is so large that the product ansatz (even in the relativised form) is not a reliable basis for calculating the Skyrme model's prediction for the long-range nuclear force. We will therefore give a heuristic derivation of the long-range force between stationary but rapidly spinning Skyrmions based on the dipole model.

Consider two triplets of well-separated and rapidly spinning scalar dipoles. The dipole moments orthogonal to the direction of the angular velocity produce a rapidly oscillating field but the dipole moment in the direction of the angular velocity produces the simple field of a static dipole, cf. (3.7). When two spinning triplets of dipoles interact the oscillating fields produce no net force but the projections of the dipole moments onto the direction of the angular velocities interact like static dipoles. According to the arguments of sect. 5 and the formulae (5.19) the potential energy for the interaction is therefore proportional to

$$
\sum_{a=1}^{3}\left(\boldsymbol{\alpha} \cdot \boldsymbol{p}_{a}\right)\left(\boldsymbol{\beta} \cdot \boldsymbol{q}_{a}\right)(\boldsymbol{\alpha} \cdot \nabla)(\boldsymbol{\beta} \cdot \nabla) \frac{1}{4 \pi R}=2 \kappa(\boldsymbol{\alpha} \cdot \mathcal{O} \boldsymbol{\beta})(\boldsymbol{\alpha} \cdot \nabla)(\boldsymbol{\beta} \cdot \nabla) \frac{1}{R} .
$$

Recalling from (3.24) that the spins for the individual Skyrmions are given by

$$
\boldsymbol{J}^{(1)}=\Lambda \boldsymbol{\alpha} \text { and } \boldsymbol{J}^{(2)}=\Lambda \boldsymbol{\beta}
$$

and the isospins by

$$
\boldsymbol{I}^{(1)}=\mathcal{A} \boldsymbol{J}^{(1)} \quad \text { and } \quad \boldsymbol{I}^{(2)}=\mathcal{B} \boldsymbol{J}^{(2)} .
$$

we can write the potential energy for the long-range interaction between spinning triplets of dipoles as

$$
C \times 2 \kappa\left(\boldsymbol{I}^{(1)} \cdot \boldsymbol{I}^{(2)}\right)\left(\boldsymbol{J}^{(1)} \cdot \nabla\right)\left(\boldsymbol{J}^{(2)} \cdot \nabla\right) \frac{1}{R}
$$


where $C$ is an unknown constant of proportionality. Remarkably this potential has exactly the same isospin and spin dependence as the zero-mass version of the one-pion exchange potential (OPEP) familiar from nuclear physics [16]. In the usual treatment of the nuclear two-body problem in the Skyrme model one projects the static two-Skyrmion potential (5.15) onto tensor products of quantum states of free Skyrmions (which model nucleons) [12]. One then also reproduces the OPEP, which is regarded as one of the successes of the Skyrme model. Here we have offered an alternative way of extracting the OPEP from the Skyrme model which takes into account the Skyrmions' spin and isospin already at the classical level. One still needs to determine the constant $C$, however. This requires more thought. In particular one needs to calculate the dipole moments of a rapidly spinning Skyrmion. Centrifugal effects could change them significantly relative to their static values.

\section{Conclusion}

In this paper we have shown that a moduli space inspired technique (the relativised product ansatz) and a point-particle model (the dipole picture) lead to the same 12-dimensional Lagrangian system for asymptotic two-Skyrmion dynamics. The interpretation of the agreement between the two approaches is that well-separated Skyrmions interact like two point-particles with suitable scalar dipole moments (adjusted to fit the asymptotic field of a single Skyrmion) not only statically but also dynamically.

Relativistic effects such as Lorentz contraction were important in our calculation, and to incorporate them we had to modify the usual moduli space prescription. In fact, on the basis of our calculations one might be surprised that in soliton theories of Bogomol'nyi type the moduli space approximation without relativistic corrections satisfactorily reproduces the results of numerical calculations in the full field theory and agrees asymptotically with point-particle approximations. The reason for this, however, is a special property of soliton theories of Bogomol'nyi type: in such theories all fields in the moduli space are at the minimum of the potential, and therefore Lorentz contraction leads to corrections of order (velocity) ${ }^{4}$. By contrast, in theories where there are static inter-soliton forces, Lorentz contraction leads to corrections of order (velocity) ${ }^{2}$, i.e. of the same order as the kinetic energy. In such theories it is therefore not consistent to include the kinetic energy in a truncated Lagrangian but to ignore relativistic effects.

While we could modify the moduli space approximation for two-Skyrmion dynamics, as proposed in [2], to take into account Lorentz-contraction of the individual Skyrmions, we have argued that it has to be abandoned altogether when studying the long-range forces between spinning Skyrmions. In its stead the dipole picture should be used to calculate those forces. Moduli space techniques are only reliable when the Skyrmions' separation and their angular speeds satisfy the

inequality (7.32) or when the Skyrmions are so close together that their interaction forces them to 
move collectively rather than individually. However, for near-coincident or coincident Skyrmions the product ansatz is no longer adequate and the more sophisticated methods of ref. [5] will be required. The Lagrangian $\tilde{L}_{2}$ would be useful in this context because it could interpolate between the moduli space and the point-particle approximation.

The immediate question that arises from our work concerns the precise nature of spinning Skyrmions. Here we have argued that the asymptotic field is that of a spinning triplet of dipoles, but it would be interesting to study spinning Skyrmions numerically to check our argument and to calculate the field near the Skyrmion's centre. Such calculations should be carried out with massive pion fields, which is necessary for the existence of a stable rotating solution of the Skyrme equations. The results would flesh out or refute the argument of sect. 9, that it is the forces between spinning Skyrmions which one needs to understand in order to calculate nuclear forces from the Skyrme model.

\section{Acknowledgements}

This paper developed partly from work done while I was a research student at DAMTP, Cambridge. I am grateful for discussions with Nick Manton and Trevor Samols during that time.

I acknowledge an SERC research assistantship.

\section{References}

[1] T.H.R. Skyrme: Proc.Roy.Soc. A260 (1961) 127

[2] N.S.Manton: Phys.Rev.Lett. 60 (1988) 1916

[3] V.B.Kopeliovich, B.E. Stern: JETP Lett. 45 (1987);

N.S.Manton: Phys.Lett. B192 (1987) 177;

J.J.M.Verbaarschot: Phys.Lett. B195 (1987) 235

[4] T.H.R. Skyrme: Nucl.Phys. 31 (1962) 556

[5] M.F. Atiyah, N.S. Manton: Phys.Lett. B222 (1989) 438; Comm.Math.Phys.153 (1993) 391

[6] D.O. Riska, E.N. Nyman: Phys.Lett. B183 (1987) 7 ;

D.O. Riska , K.Dannbom: Physica Scripta 37 (1988) 7

[7] B.Shao, N.R.Walet,R.D.Amado: Inertial Parameters of the Skyrmion-Skyrmion System with the Product Ansatz. University of Pennsylvania preprint UPR-0128MT (1993)

[8] N.S. Manton: Phys.Lett. 154B (1985) 397

[9] G.W. Gibbons, P.J. Ruback: Phys.Rev.Lett. 57 (1986) 1492 
[10] G.S.Adkins, C.R.Nappi, E.Witten: Nucl.Phys. B228 (1983) 552

[11] G.Holzwarth, B.Schwesinger: Rep.Prog.Phys.49 (1986) 825

[12] A.Jackson, A.D.Jackson: Nucl.Phys. A432 (1985) 567 ;

R.VinhMau et al.: Phys.Lett. B150 (1985) 259

[13] G.S.Adkin, C.R.Nappi: Nucl.Phys. B233 (1984) 109

[14] J.J.M.Verbaarschot, T.S.Walhout, J.Wambach, H.W.Wyld: Nucl.Phys. A468 (1987) 520

[15] T.S.Walhout, J.Wambach: Phys.Rev.Lett. 67 (1991) 314

[16] T. Ericson , W.Weise: Pions and Nuclei. Oxford: Oxford University Press 1988 